SISSA 59/2005/EP

IC $/ 2005 / 045$

hep-ph/0508243

\title{
Majorana CP-Violating Phases, RG Running of Neutrino Mixing Parameters and Charged Lepton Flavour Violating Decays
}

\author{
S. T. Petcov ${ }^{a, b) *}$ T. Shindou ${ }^{a, b) \dagger}$, Y. Takanishi ${ }^{c) \ddagger}$, \\ a) Scuola Internazionale Superiore di Studi Avanzati, I-34014 Trieste, Italy \\ b) Istituto Nazionale di Fisica Nucleare, Sezione di Trieste, I-34014 Trieste, Italy \\ c) The Abdus Salam International Centre for Theoretical Physics, Strada Costiera 11, \\ I-34100 Trieste, Italy
}

\begin{abstract}
We consider the MSSM with see-saw mechanism of neutrino mass generation and soft SUSY breaking with flavour-universal boundary conditions at the GUT scale, in which the lepton flavour violating (LFV) decays $\mu \rightarrow e+\gamma, \tau \rightarrow \mu+\gamma$, etc., are predicted with rates that can be within the reach of present and planned experiments. These predictions depend critically on the matrix of neutrino Yukawa couplings $\mathbf{Y}_{\nu}$ which can be expressed in terms of the light and heavy right-handed $(\mathrm{RH})$ neutrino masses, neutrino mixing matrix $U_{\text {PMNS }}$, and an orthogonal matrix $\mathbf{R}$. We investigate the effects of Majorana CP-violation phases in $U_{\mathrm{PMNS}}$, and of the $\mathrm{RG}$ running of light neutrino masses and mixing angles from $M_{Z}$ to the $\mathrm{RH}$ Majorana neutrino mass scale $M_{R}$, on the predictions for the rates of LFV decays $\mu \rightarrow e+\gamma, \tau \rightarrow \mu+\gamma$ and $\tau \rightarrow e+\gamma$. Results for neutrino mass spectrum with normal hierarchy, values of the lightest $\nu$-mass in the range $0 \leq m_{1} \leq 0.30 \mathrm{eV}$, and quasi-degenerate heavy $\mathrm{RH}$ Majorana neutrinos in the cases of $\mathbf{R}=\mathbf{1}$ and complex matrix $\mathbf{R}$ are presented. We find that the effects of the Majorana $\mathrm{CP}$-violation phases and of the $\mathrm{RG}$ evolution of neutrino mixing parameters can change by few orders of magnitude the predicted rates of the LFV decays $\mu \rightarrow e+\gamma$ and $\tau \rightarrow e+\gamma$. The impact of these effects on the $\tau \rightarrow \mu+\gamma$ decay rate is typically smaller and only possible for $m_{1} \gtrsim 0.10 \mathrm{eV}$. If the RG running effects are negligible, in a large region of soft SUSY breaking parameter space the ratio of the branching ratios of the $\mu \rightarrow e+\gamma$ and $\tau \rightarrow e+\gamma(\tau \rightarrow \mu+\gamma)$ decays is entirely determined in the case of $\mathbf{R} \cong \mathbf{1}$ by the values of the neutrino mixing parameters at $M_{Z}$.
\end{abstract}

October 31, 2018

*Also at: Institute of Nuclear Research and Nuclear Energy, Bulgarian Academy of Sciences, 1784 Sofia, Bulgaria

${ }^{\dagger}$ E-mail: shindou@he.sissa.it

‡E-mail: yasutaka@ictp.trieste.it 


\section{Introduction}

The experiments with solar, atmospheric, reactor and accelerator neutrinos [1-5] have provided during the last several years compelling evidence for the existence of neutrino oscillations caused by nonzero neutrino masses and neutrino mixing. The $\nu$-oscillation data obtained in the solar neutrino, atmospheric neutrino, KamLAND (KL) and K2K experiments imply the existence of $3-\nu$ mixing in the weak charged-lepton current (see, e.g., [6]):

$$
\nu_{l \mathrm{~L}}=\sum_{j=1}^{3} U_{l j} \nu_{j \mathrm{~L}}, \quad l=e, \mu, \tau,
$$

where $\nu_{l L}$ are the flavour neutrino fields, $\nu_{j \mathrm{~L}}$ is the field of neutrino $\nu_{j}$ having a mass $m_{j}$ and $U$ is the Pontecorvo-Maki-Nakagawa-Sakata (PMNS) mixing matrix [7], $U \equiv U_{\text {PMNS }}$. It follows from the results of the neutrino oscillation and ${ }^{3} \mathrm{H} \beta$-decay experiments [8] that the neutrino masses $m_{j}$ are much smaller than the masses of the charged leptons and quarks: $m_{j}<2.3 \mathrm{eV}(95 \% \text { C.L. })^{1}$.

A natural explanation of the smallness of neutrino masses is provided by the see-saw mechanism of neutrino mass generation [11]. The see-saw mechanism predicts the light massive neutrinos $\nu_{j}$ to be Majorana particles. An integral part of the mechanism are the heavy right-handed $(\mathrm{RH})$ Majorana neutrinos. Being singlets with respect to the Standard Theory gauge symmetry group [12], the RH neutrinos can acquire Majorana masses which are much larger than the electroweak symmetry breaking scale. The CP-violating decays of the heavy RH Majorana neutrinos in the Early Universe could generate, through the leptogenesis scenario, the observed baryon asymmetry of the Universe [13].

In grand unified theories (GUT) the masses of the heavy RH Majorana neutrinos are typically by a few to several orders of magnitude smaller than the scale of unification of the electroweak and strong interactions, $M_{\mathrm{GUT}} \sim 2 \times 10^{16} \mathrm{GeV}$. The presence of the electroweak symmetry breaking scale and of the scale of RH neutrino masses $M_{R}$ in a theory ${ }^{2}$ can lead to a hierarchy problem. In SUSY (GUT) theories this problem could be avoided: the hierarchy between the two mass (energy) scales is stabilised. Thus, the SUSY (GUT) theories with seesaw mechanism provide an internally consistent framework for generation of light neutrino masses $m_{j}$ and of the baryon asymmetry of the Universe.

The existence of the flavour neutrino mixing, eq. (11), implies that the individual lepton charges, $L_{l}, l=e, \mu, \tau$, are not conserved (see, e.g., [14]). Thus, lepton charge non-conserving processes like $\mu^{-} \rightarrow e^{-}+\gamma, \mu^{-} \rightarrow e^{-}+e^{+}+e^{-}, \tau^{-} \rightarrow e^{-}+\gamma, \tau^{-} \rightarrow \mu^{-}+\gamma, \mu^{-}+(A, Z) \rightarrow$ $e^{-}+(A, Z)$, etc. are allowed and should take place. However, if the neutrino mixing in the weak charged lepton current, eq. (11), is the only source of $L_{e}, L_{\mu}$ and $L_{\tau}$ non-conservation, as in the minimally extended Standard Theory with massive neutrinos [15], the rates and cross sections of the indicated lepton flavour violating (LFV) processes are suppressed by the factor [15] (see also [16]) $\left(m_{j} / M_{W}\right)^{4}<6.7 \times 10^{-43}, M_{W}$ being the $W^{ \pm}$mass. This renders

\footnotetext{
${ }^{1}$ More stringent upper limit on $m_{j}$ follows from the constraints on the sum of neutrino masses obtained from cosmological/astrophysical observations, namely, the CMB data of the WMAP experiment combined with data from large scale structure surveys (2dFGRS, SDSS) [9]: $\sum_{j} m_{j}<(0.7-2.0) \mathrm{eV}$ (95\% C.L.), where we have included a conservative estimate of the uncertainty in the upper limit (see, e.g., [10]).

${ }^{2}$ In the case of three heavy RH Majorana neutrinos $N_{j}$ with masses $M_{j}$ we will consider, $M_{R}$ is determined by the mass of the lightest RH Majorana neutrino, $M_{R}=\min \left(M_{j}\right)$.
} 
them unobservable. Indeed, the existing upper limits on, e.g. $\mu \rightarrow e+\gamma, \mu \rightarrow 3 e$, and $\tau \rightarrow \mu+\gamma$ decay branching ratios and on the relative cross section of the $\mu-e$ conversion process $\mu^{-}+\mathrm{Ti} \rightarrow e^{-}+\mathrm{Ti}$, are at least by 30 orders of magnitude bigger [17-19] (90\% C.L.):

$$
\begin{gathered}
\operatorname{BR}(\mu \rightarrow e+\gamma)<1.2 \times 10^{-11}, \quad \operatorname{BR}(\mu \rightarrow 3 e)<1.2 \times 10^{-12}, \\
\operatorname{BR}(\tau \rightarrow \mu+\gamma)<6.8 \times 10^{-8}, \quad \mathrm{R}\left(\mu^{-}+\mathrm{Ti} \rightarrow e^{-}+\mathrm{Ti}\right)<4.3 \times 10^{-12} .
\end{gathered}
$$

Future experiments with increased sensitivity can reduce the current bounds on $\mathrm{BR}(\mu \rightarrow$ $e+\gamma), \operatorname{BR}(\tau \rightarrow \mu+\gamma)$ and on $\mathrm{R}\left(\mu^{-}+(A, Z) \rightarrow e^{-}+(A, Z)\right)$ by a few orders of magnitude (see, e.g., [20]). In the experiment MEG under preparation at PSI [21] it is planned to reach a sensitivity to

$$
\mathrm{BR}(\mu \rightarrow e+\gamma) \sim\left(10^{-13}-10^{-14}\right) .
$$

It has been noticed in 1986 that in SUSY (GUT) theories with see-saw mechanism of neutrino mass generation, the rates and cross sections of the LFV processes can be strongly enhanced [22] and could be close to existing upper limits. If the SUSY breaking occurs via soft terms with universal boundary conditions at a scale $M_{X}$ above the RH Majorana neutrino mass scale ${ }^{3} M_{R}, M_{X}>M_{R}$, the renormalisation group effects transmit the LFV from the neutrino mixing at $M_{X}$ to the effective mass terms of the scalar leptons at $M_{R}$, generating new LFV corrections to the flavour-diagonal mass terms. For scalar lepton masses of $\sim$ few hundred $\mathrm{GeV}$, the LFV mass corrections at $M_{R}$ are typically of the order of a few $\mathrm{GeV}$ and thus are much larger than the light neutrino masses $m_{j}$. As a consequence (and in contrast to the non-supersymmetric case), the LFV scalar lepton mixing at $M_{R}$ generates additional contributions to the amplitudes of the LFV decays and reactions which are not suppressed by the small values of neutrino masses $m_{j}$. As a result, the LFV processes can proceed with rates and cross sections which are within the sensitivity of presently operating and future planned experiments $[22,24]$ (see also, e.g., [25-31]).

One of the basic ingredients of the see-saw mechanism is the matrix of neutrino Yukawa couplings, $\mathbf{Y}_{\nu}$. Leptogenesis depends on $\mathbf{Y}_{\nu}$ as well. In the large class of SUSY models with see-saw mechanism of neutrino mass generation and flavour-universal soft SUSY breaking at a scale $M_{X}>M_{R}$, we will consider, the probabilities of LFV processes also depend strongly on $\mathbf{Y}_{\nu}$ (see, e.g., $[25,26]$ ). Thus, the matrix of neutrino Yukawa couplings $\mathbf{Y}_{\nu}$ connects in the see-saw theories the light neutrino mass generation with leptogenesis; in SUSY theories with soft flavour-universal SUSY breaking at $M_{X}>M_{R}, \mathbf{Y}_{\nu}$ links the light neutrino mass generation and leptogenesis with LFV processes (see, e.g., [28,29]).

The matrix $\mathbf{Y}_{\nu}$ can be expressed in terms of the light neutrino and heavy $\mathrm{RH}$ neutrino masses, the neutrino mixing matrix $U_{\mathrm{PMNS}}$, and an orthogonal matrix $\mathbf{R}$ [25]. Leptogenesis can take place only if $\mathbf{R}$ is complex. Obviously, $\mathbf{Y}_{\nu}$ depends on the Majorana phases in the PMNS matrix $U_{\text {PMNS. }}$ It was shown in [28] (see also [26]) that in the case of $\mathbf{R} \neq \mathbf{1}$, the Majorana CP-violation phases in $U_{\text {PMNS }}$ [32] can affect significantly the predictions for the rates of LFV decays $\mu \rightarrow e+\gamma, \tau \rightarrow \mu+\gamma$, etc. in the class of supersymmetric theories of interest.

The matrix $\mathbf{Y}_{\nu}$ can be defined, strictly speaking, only at scales not smaller than $M_{R}$. The probabilities of LFV processes depend on $\mathbf{Y}_{\nu}$ at the scale $M_{R}, \mathbf{Y}_{\nu}=\mathbf{Y}_{\nu}\left(M_{R}\right)$. In order

\footnotetext{
${ }^{3}$ The possibility of "flavour-blind" SUSY breaking of interest is realised, e.g. in gravity-mediated SUSY breaking scenarios (see, e.g., [23]).
} 
to evaluate $\mathbf{Y}_{\nu}\left(M_{R}\right)$ one has to know, in particular, the light neutrino masses $m_{j}$ and the mixing matrix $U_{\text {PMNS }}$ at $M_{R}$, i.e., one has to take into account the renormalisation group (RG) "running" of $m_{j}$ and $U_{\text {PMNS }}$ from the scale $M_{Z} \sim 100 \mathrm{GeV}$, at which the neutrino mixing parameters are measured, to the scale $M_{R}[33-37]$.

Working in the framework of the class of SUSY theories with see-saw mechanism and flavour-universal soft SUSY breaking at a scale $M_{X}>M_{R}$, we investigate in the present article the effects of RG running of neutrino mixing parameters and of the Majorana CPviolation phases on the predictions for the rates charged lepton flavour violating decays. We consider neutrino mass spectrum with normal hierarchy $(\mathrm{NH}), m_{1}<m_{2}<m_{3}$, allowing the lightest neutrino mass $m_{1}$ to vary in the range $0 \leq m_{1} \leq 0.30 \mathrm{eV}$. The latter covers all possible varieties of light neutrino mass spectrum of the NH type: normal hierarchical, quasi-degenerate as well as spectrum with partial normal hierarchy (see, e.g., [6]). In order to find the changes of the neutrino masses $m_{j}$ and mixing angles in $U_{\text {PMNs }}$ due to the RG evolution from the scale $M_{Z} \sim 100 \mathrm{GeV}$ to $M_{R}$, the corresponding 1-loop RG equations for the see-saw Majorana mass matrix of the left-handed neutrinos were solved numerically. The best fit values of the known solar and atmospheric neutrino mixing angles and neutrino mass squared differences at $M_{Z}$ were used as "initial conditions" for the solution of the RG equations. The values of the neutrino mixing parameters at the scale $M_{R}$ served as input in the calculation of $\mathbf{Y}_{\nu}\left(M_{R}\right)$ and correspondingly of the rates of the LFV decays $l_{i} \rightarrow l_{j}+\gamma$. The latter were performed for quasi-degenerate heavy RH Majorana neutrinos for a number of typical sets of values of the soft SUSY breaking parameters. We have obtained results both in the cases of $\mathbf{R}=\mathbf{1}$ and of complex matrix $\mathbf{R}$.

Detailed predictions for the rates of the LFV processes in the class of SUSY models with see-saw mechanism considered were obtained, e.g. in refs. [25-31]. However, our study overlaps little with those performed in these articles.

\section{Neutrino Mixing Parameters from Neutrino Oscillation Data}

We will use the standard parametrisation of the PMNS matrix $U_{\mathrm{PMNS}}$ (see, e.g., [38]):

$$
U_{\mathrm{PMNS}}=\left(\begin{array}{ccc}
c_{12} c_{13} & s_{12} c_{13} & s_{13} e^{-i \delta} \\
-s_{12} c_{23}-c_{12} s_{23} s_{13} e^{i \delta} & c_{12} c_{23}-s_{12} s_{23} s_{13} e^{i \delta} & s_{23} c_{13} \\
s_{12} s_{23}-c_{12} c_{23} s_{13} e^{i \delta} & -c_{12} s_{23}-s_{12} c_{23} s_{13} e^{i \delta} & c_{23} c_{13}
\end{array}\right) \operatorname{diag}\left(1, e^{i \frac{\alpha}{2}}, e^{i \frac{\beta_{M}}{2}}\right),
$$

where $c_{i j}=\cos \theta_{i j}, s_{i j}=\sin \theta_{i j}$, the angles $\theta_{i j}=[0, \pi / 2], \delta=[0,2 \pi]$ is the Dirac CPviolating phase and $\alpha$ and $\beta_{M}$ are two Majorana CP-violation phases [32,39]. One can identify the neutrino mass squared difference responsible for solar neutrino oscillations, $\Delta m_{\odot}^{2}$, with $\Delta m_{21}^{2} \equiv m_{2}^{2}-m_{1}^{2}, \Delta m_{\odot}^{2}=\Delta m_{21}^{2}>0$. The neutrino mass squared difference driving the dominant $\nu_{\mu} \rightarrow \nu_{\tau}\left(\bar{\nu}_{\mu} \rightarrow \bar{\nu}_{\tau}\right)$ oscillations of atmospheric $\nu_{\mu}\left(\bar{\nu}_{\mu}\right)$ is then given by $\left|\Delta m_{\mathrm{A}}^{2}\right|=\left|\Delta m_{31}^{2}\right| \cong\left|\Delta m_{32}^{2}\right| \gg \Delta m_{21}^{2}$. The corresponding solar and atmospheric neutrino mixing angles, $\theta_{\odot}$ and $\theta_{\mathrm{A}}$, coincide with $\theta_{12}$ and $\theta_{23}$, respectively. The angle $\theta_{13}$ is limited by the data from the CHOOZ and Palo Verde experiments [40].

The existing neutrino oscillation data allow us to determine $\Delta m_{21}^{2},\left|\Delta m_{31}^{2}\right|, \sin ^{2} \theta_{12}$ and $\sin ^{2} 2 \theta_{23}$ with a relatively good precision and to obtain rather stringent limits on $\sin ^{2} \theta_{13}$ 
(see, e.g., $[41,42]$ ). The best fit values and the $95 \%$ C.L. allowed ranges of $\Delta m_{21}^{2}, \sin ^{2} \theta_{12}$, $\left|\Delta m_{31}^{2}\right|$ and $\sin ^{2} 2 \theta_{23}$ read ${ }^{4}$.

$$
\begin{gathered}
\Delta m_{21}^{2}=8.0 \times 10^{-5} \mathrm{eV}^{2}, \quad \sin ^{2} \theta_{21}=0.31, \\
\Delta m_{21}^{2}=(7.3-8.5) \times 10^{-5} \mathrm{eV}^{2}, \quad \sin ^{2} \theta_{12}=(0.26-0.36), \\
\left|\Delta m_{31}^{2}\right|=2.2 \times 10^{-3} \mathrm{eV}^{2}, \quad \sin ^{2} 2 \theta_{23}=1.0, \\
\left|\Delta m_{31}^{2}\right|=(1.7-2.9) \times 10^{-3} \mathrm{eV}^{2}, \quad \sin ^{2} 2 \theta_{23} \geq 0.90 .
\end{gathered}
$$

A combined 3- $\nu$ oscillation analysis of the solar neutrino, KL and CHOOZ data gives $[41,42]$

$$
\sin ^{2} \theta_{13}<0.027(0.047), \quad \text { at } 95 \%(99.73 \%) \text { C.L. }
$$

The neutrino oscillation parameters discussed above can (and very likely will) be measured with much higher accuracy in the future (see, e.g., [6]).

The sign of $\Delta m_{\mathrm{A}}^{2}=\Delta m_{31}^{2}$, as it is well known, cannot be determined from the present (SK atmospheric neutrino and $\mathrm{K} 2 \mathrm{~K})$ data. The two possibilities, $\Delta m_{31(32)}^{2}>0$ or $\Delta m_{31(32)}^{2}<0$ correspond to two different types of $\nu$-mass spectrum:

- with normal hierarchy $m_{1}<m_{2}<m_{3}, \Delta m_{\mathrm{A}}^{2}=\Delta m_{31}^{2}>0$, and

- with inverted hierarchy $m_{3}<m_{1}<m_{2}, \Delta m_{\mathrm{A}}^{2}=\Delta m_{32}^{2}<0$.

Depending on the sign of $\Delta m_{\mathrm{A}}^{2}, \operatorname{sgn}\left(\Delta m_{\mathrm{A}}^{2}\right)$, and the value of the lightest neutrino mass, $\min \left(m_{j}\right)$, the $\nu$-mass spectrum can be

- Normal Hierarchical: $m_{1} \ll m_{2} \ll m_{3}, m_{2} \cong\left(\Delta m_{\odot}^{2}\right)^{\frac{1}{2}} \sim 0.009 \mathrm{eV}, m_{3} \cong\left|\Delta m_{\mathrm{A}}^{2}\right|^{\frac{1}{2}} \sim 0.045 \mathrm{eV}$;

- Inverted Hierarchical: $m_{3} \ll m_{1}<m_{2}$, with $m_{1,2} \cong\left|\Delta m_{\mathrm{A}}^{2}\right|^{\frac{1}{2}} \sim 0.045 \mathrm{eV}$;

- Quasi-Degenerate $(Q D): m_{1} \cong m_{2} \cong m_{3} \cong m_{0}, m_{j}^{2} \gg\left|\Delta m_{\mathrm{A}}^{2}\right|, m_{0} \gtrsim 0.10 \mathrm{eV}$.

It is well-known, the theories employing the see-saw mechanism of neutrino mass generation [11] we are going to discuss further, predict the massive neutrinos $\nu_{j}$ to be Majorana particles. Determining the nature of massive neutrinos is one of the most formidable and pressing problems in today's neutrino physics. Being of fundamental importance for understanding the origin of $\nu$-masses and mixing, it is widely recognised as a highest priority goal of the future studies of neutrino mixing (see, e.g., $[6,45]$ ).

If the massive neutrinos $\nu_{j}$ are Majorana fermions, getting information about the Majorana $\mathrm{CP}$-violation phases in $U_{\mathrm{PMNS}}$ would be highly problematic. The oscillations of flavour neutrinos, $\nu_{l} \rightarrow \nu_{l^{\prime}}$ and $\bar{\nu}_{l} \rightarrow \bar{\nu}_{l^{\prime}}, l, l^{\prime}=e, \mu, \tau$, are insensitive to the Majorana CP-violation phases $\alpha$ and $\beta_{M}[32,46]$; they are insensitive to the nature - Dirac or Majorana, of massive neutrinos $\nu_{j}$. The only feasible experiments that at present have the potential of establishing the Majorana nature of light neutrinos $\nu_{j}$ and of providing information on the Majorana CP-violation phases in $U_{\text {PMNS }}$ are the experiments searching for the neutrinoless double beta $\left((\beta \beta)_{0 \nu^{-}}\right)$decay, $(A, Z) \rightarrow(A, Z+2)+e^{-}+e^{-}$(see, e.g., $\left.[14,45,47]\right)$. If the $(\beta \beta)_{0 \nu^{-} \text {decay is }}$ observed, the measurement of the $(\beta \beta)_{0 \nu}$-decay half-life combined with information on the absolute scale of neutrino masses (or on $\min \left(m_{j}\right)$ ), might allow to significantly constrain, or possibly determine, the Majorana phase $\alpha$ [38,48], for instance. Establishing CP-violation due to the Majorana phases in $U_{\mathrm{PMNS}}$, however, was found to be remarkably challenging [49].

\footnotetext{
${ }^{4}$ The data imply, in particular, that the high-LMA solution (see, e.g., [43]) is excluded at $\sim 3.3 \sigma$. Maximal solar neutrino mixing is ruled out at $\sim 6 \sigma$; at $95 \%$ C.L. one finds $\cos 2 \theta_{\odot} \geq 0.28$ [41], which has important implications [44].
} 


\section{The See-Saw Mechanism and Neutrino Yukawa Couplings}

We consider the minimal supersymmetric standard model with RH neutrinos (MSSMRN). The superpotential for the lepton sector is given by

$$
\mathcal{W}_{l}=\hat{E}_{i}^{c}\left(\mathbf{Y}_{\mathrm{E}}\right)_{i j} \hat{L}_{j} \cdot \hat{H}_{d}+\hat{N}_{i}^{c}\left(\mathbf{Y}_{\nu}\right)_{i j} \hat{L}_{j} \cdot \hat{H}_{u}-\frac{1}{2}\left(\mathbf{M}_{\mathrm{N}}\right)_{i j} \hat{N}_{i}^{c} \hat{N}_{j}^{c}
$$

where $\hat{L}_{j}, \hat{E}_{j}^{c}$ and $\hat{N}_{j}^{c}, j=e, \mu, \tau \equiv 1,2,3$, are the chiral supermultiplets respectively of the $S U(2)_{L}$ doublet lepton field $L_{j L}$ and of the $S U(2)_{L}$ singlet charged lepton and neutrino fields $l_{j L}^{c} \equiv C\left(\bar{l}_{j R}\right)^{T}$ and $N_{j L}^{c} \equiv C \bar{N}_{j R}^{T}$, where $l_{j R}$ and $N_{j R}$ are RH fields and $C$ is the charge conjugation matrix, while $\hat{H}_{u}$ and $\hat{H}_{d}$ are the supermultiplets of the two Higgs doublet fields $H_{u}$ and $H_{d}$ carrying weak hypercharges $-\frac{1}{2}$ and $\frac{1}{2}$. In eq. (8), $\mathbf{Y}_{\mathrm{E}}$ is the $3 \times 3$ matrix of the Yukawa couplings of the charged leptons, $\mathbf{Y}_{\nu}$ is the $3 \times 3$ matrix of neutrino Yukawa couplings, and $\mathbf{M}_{\mathrm{N}}$ is the Majorana mass matrix of the $\mathrm{RH}$ neutrinos $N_{j R}$.

In the framework of MSSMRN one can always choose a basis in which both $\mathbf{Y}_{\mathrm{E}}$ and $\mathbf{M}_{\mathrm{N}}$ are diagonal. Henceforth, we will work in that basis and will denote by $\mathbf{D}_{\mathrm{N}}$ the corresponding diagonal RH neutrino mass matrix, $\mathbf{D}_{\mathrm{N}}=\operatorname{diag}\left(M_{1}, M_{2}, M_{3}\right)$.

Below the see-saw scale, $M_{R}=\min \left(M_{j}\right)$, the singlet supermultiplets $\hat{N}_{i}^{c}$ containing the $\mathrm{RH}$ neutrino fields are integrated out, and the superpotential, eq. (8), becomes

$$
\mathcal{W}_{l}=\hat{E}_{i}^{c}\left(\mathbf{Y}_{\mathrm{E}}\right)_{i j} \hat{L}_{j} \cdot \hat{H}_{d}-\frac{1}{2}\left(K_{N}\right)^{i j}\left(\hat{L}_{i} \cdot \hat{H}_{u}\right)\left(\hat{L}_{j} \cdot \hat{H}_{u}\right)
$$

where

$$
\left(K_{N}\right)^{i j}=\left(Y_{\nu}^{T}\right)^{i k}\left(M_{\mathrm{N}}^{-1}\right)^{k l}\left(Y_{\nu}\right)^{l j}
$$

The last term yields Majorana mass term for the left-handed (LH) flavour neutrinos after the electroweak symmetry breaking,

$$
\mathcal{L}_{m}^{\nu}=-\frac{1}{2} \bar{\nu}_{R j}^{C}\left(m_{\nu}\right)^{j k} \nu_{L k}+\text { h.c. }
$$

where $\nu_{R j}^{C} \equiv C\left(\bar{\nu}_{L j}\right)^{T}$ and

$$
\left(m_{\nu}\right)^{i j}=\left(K_{N}\right)^{i j}\left\langle H_{u}\right\rangle^{2}
$$

Here $\left\langle H_{u}\right\rangle$ is the vacuum expectation value of $H_{u},\left\langle H_{u}\right\rangle \equiv v_{u}=v \sin \beta$, where $v=174 \mathrm{GeV}$. The neutrino mass matrix is related to the light neutrino masses $m_{j}$ and the PMNS mixing matrix as

$$
\left(m_{\nu}\right)^{i j}=\left(U^{*}\right)^{i k} m_{k}\left(U^{\dagger}\right)^{k j}
$$

Using (12) and (13), we can rewrite the matching condition, eq. (10), at the energy scale $M_{R}$ in the form

$$
\mathbf{U}^{*}\left(M_{R}\right) \mathbf{D}_{\nu}\left(M_{R}\right) \mathbf{U}^{\dagger}\left(M_{R}\right)=-v_{u}^{2} \mathbf{Y}_{\nu}^{T}\left(M_{R}\right) \mathbf{M}_{\mathrm{N}}^{-1}\left(M_{R}\right) \mathbf{Y}_{\nu}\left(M_{R}\right)
$$


where $\mathbf{D}_{\nu}=\operatorname{diag}\left(m_{1}, m_{2}, m_{3}\right)$. Thus, in the basis in which the RH neutrino mass matrix is diagonal $\mathbf{M}_{\mathrm{N}}=\mathbf{D}_{\mathrm{N}}$, the neutrino Yukawa coupling at $M_{R}$ can be parametrised as [25]

$$
\mathbf{Y}_{\nu}\left(M_{R}\right)=\frac{1}{v_{u}} \sqrt{\mathbf{D}_{N}\left(M_{R}\right)} \mathbf{R} \sqrt{\mathbf{D}_{\nu}\left(M_{R}\right)} \mathbf{U}^{\dagger}\left(M_{R}\right)
$$

Here $\mathbf{R}$ is a complex orthogonal matrix, $\mathbf{R}^{T} \mathbf{R}=\mathbf{1}$. The PMNS matrix $\mathbf{U}$ and the light neutrino masses in $\mathbf{D}_{\nu}$ in eq. (15) should be evaluated at $M_{R}$ with the help of the renormalisation group equations which describe their evolution from the the scale $M_{Z}$, where they are measured ${ }^{5}$, to the scale $M_{R}$.

\section{The Renormalisation Group Evolution Effects}

The RG running corrections to the neutrino mass matrix in MSSM have been studied by many authors (see, e.g., $[36,37]$ and the articles quoted therein). In order to find the RG evolution from $M_{Z}$ to $M_{R}$ of the neutrino mixing angles $\theta_{12}, \theta_{23}, \theta_{13}$ and the masses $m_{j}$, we have solved numerically the relevant 1-loop RG equation for the neutrino mass matrix, eq. (12), in MSSMRN [33] (see also, e.g., [30,36,37]). The case of neutrino mass spectrum with normal hierarchy at $M_{Z}, m_{1}\left(M_{Z}\right)<m_{2}\left(M_{Z}\right)<m_{3}\left(M_{Z}\right)$, was considered. The best fit values of the neutrino oscillation parameters $\theta_{12}, \theta_{23}, \Delta m_{21}^{2}$ and $\Delta m_{31}^{2}$ at $M_{Z}$, given in eqs. (5) and (6), were used as input in the calculation of the RG running effects. The CHOOZ mixing angle at $M_{Z}$ was set to zero, $\sin \theta_{13}\left(M_{Z}\right)=0$.

We will summarise below some of the results regarding the RG running of the neutrino mixing parameters, which are relevant for our further analysis.

- The corrections can become significant for relatively large $\tan \beta$.

- The mixing angles are stable with respect to the RG running in the case of normal hierarchical neutrino mass spectrum, $m_{1} \ll m_{2} \ll m_{3}$.

- For $m_{1} \gtrsim 0.05 \mathrm{eV}$, the RG running effects can change significantly the neutrino (lepton) mixing angles provided $\tan \beta$ is sufficiently large.

- The solar neutrino mixing angle $\theta_{\odot} \equiv \theta_{12}$ at $M_{R}$ depends strongly on the Majorana phase $\alpha$, which plays very important role in the predictions of the effective Majorana mass in $(\beta \beta)_{0 \nu}$-decay (see, e.g. [47]). The effect of RG running for $\theta_{12}$ is largest when $\alpha$ takes the $\mathrm{CP}$-conserving value $\alpha=0$; it is relatively small or negligible for the alternative CP-conserving values of $\alpha$ [50], $\alpha= \pm \pi$. For $\alpha=0$ and $\tan \beta \sim 50$, for instance, we have $\tan ^{2} \theta_{12}\left(M_{R}\right) \lesssim 0.5 \tan ^{2} \theta_{12}\left(M_{Z}\right)$ for $m_{1} \gtrsim 0.02 \mathrm{eV}$.

- The RG running effects depend weakly on the Majorana phase $\beta_{M}$.

- The atmospheric neutrino mixing angle $\theta_{\mathrm{A}} \equiv \theta_{23}$ is essentially stable against the RG running corrections, except in the cases when $m_{1}$ and/or $\tan \beta$ are/is relatively large, i.e., (i) when $m_{1} \gtrsim 0.2 \mathrm{eV}$ and $\alpha \lesssim 3 \pi / 4$ if $\tan \beta \sim 10$, and (ii) for any $m_{1}$ and $\alpha$ if $\tan \beta \gtrsim 40$.

\footnotetext{
${ }^{5}$ Strictly speaking, the neutrino mixing parameters are measured at scales $\sim 1 \mathrm{GeV}$ and $\sim 1 \mathrm{MeV}$ which are smaller than $M_{Z}$. However, these physical parameters do not get any significant corrections from the RG running below $M_{Z}$.
} 
- The correction to $\sin \theta_{13}$ is relatively small even for large $\tan \beta$ if $m_{1} \lesssim 0.05 \mathrm{eV}$, and for any $m_{1} \gtrsim 0.30 \mathrm{eV}$ if $\theta_{13}\left(M_{Z}\right) \cong 0$ and $\alpha \cong 0$. For $\alpha=\pi$ and $\tan \beta \sim 50$ one can have $\sin \theta_{13}\left(M_{R}\right) \gtrsim 0.10$ for $m_{1} \gtrsim 0.08 \mathrm{eV}$ even if $\sin \theta_{13}\left(M_{Z}\right)=0$. If, e.g. $\sin \theta_{13}\left(M_{Z}\right)=0.10$ $\mathrm{eV}$, the RG effects lead for $\alpha=0(\pi / 2 \lesssim \alpha \lesssim \pi)$ to considerably smaller (larger) values of $\sin \theta_{13}\left(M_{R}\right)$ at $m_{1} \gtrsim 0.05 \mathrm{eV}$; for $\alpha \sim \pi / 4$ one has $\sin \theta_{13}\left(M_{Z}\right) \cong \sin \theta_{13}\left(M_{R}\right)$.

- For sufficiently large $\tan \beta, \tan \beta \gtrsim 30$, the value of $\Delta m_{21}^{2}\left(M_{R}\right)$ depends strongly on $m_{1}$ in the interval $m_{1} \gtrsim 0.05 \mathrm{eV}$, and on $\alpha$ for $m_{1} \gtrsim 0.10 \mathrm{eV}$. In the case of $\tan \beta \sim$ 50 , for instance, we have $\Delta m_{21}^{2}\left(M_{R}\right) \cong 3 \Delta m_{21}^{2}\left(M_{Z}\right)\left(\Delta m_{21}^{2}\left(M_{R}\right) \cong 10 \Delta m_{21}^{2}\left(M_{Z}\right)\right)$ at $m_{1}=0.05(0.10) \mathrm{eV}$. The dependence of $\Delta m_{31}^{2}\left(M_{R}\right)$ on $m_{1}$ and $\alpha$ is rather weak, unless $\tan \beta \gtrsim 40$ and $m_{1} \gtrsim 0.10 \mathrm{eV}$.

- Some products of the neutrino mixing parameters, such as $s_{12} c_{12} c_{23}\left(m_{1}-m_{2} e^{i \alpha}\right)$ are practically stable with respect to RG running (for further discussion see Appendix A and, e.g. refs. $[30,36,37])$.

Similar results hold also in the case of neutrino mass spectrum with inverted hierarchy.

The RG equation for the neutrino mass matrix in MSSMRN and its approximate solution as well as analytic expressions for some of the neutrino mixing parameters at $M_{R}$ in the case of interest are given in Appendix A. The specific features of the effects of RG running of the neutrino mixing angles and mass (squared) differences discussed above are illustrated in Figs. 1 and 2, where the values of $\tan ^{2} \theta_{12}, \tan ^{2} \theta_{23}$ and $\sin \theta_{13}$, and of $\left(m_{2}-m_{1}\right), \Delta m_{21}^{2}$, $\left(m_{3}-m_{1}\right)$ and $\Delta m_{31}^{2}$, at $M_{R}=2 \times 10^{13} \mathrm{GeV}$ are shown as functions of $\min \left(m_{j}\right)=m_{1}$. The neutrino mass spectrum is assumed to be with normal hierarchy at $M_{Z}$. The two figures correspond to the following values of the neutrino oscillation parameters and $\beta_{M}$ at $M_{Z}$ : $\tan ^{2} \theta_{12}=0.40, \tan ^{2} \theta_{23}=1, \Delta m_{21}^{2}=8.0 \times 10^{-5} \mathrm{eV}^{2}, \Delta m_{31}^{2}=2.2 \times 10^{-3} \mathrm{eV}^{2}, \sin \theta_{13}=0$ and $\beta_{M}=0$.

The results obtained in the present Section are used in the calculations of the $l_{i} \rightarrow l_{j}+\gamma$ decay rates to which we turn next.

\section{Lepton Flavour Violation in MSSMRN and $l_{i} \rightarrow l_{j}+\gamma$ Decays}

In the MSSMRN under discussion, the boundary conditions for the soft SUSY breaking are assumed to take place at some scale $M_{X}>M_{R}$, typically at $M_{X} \gtrsim M_{\mathrm{GUT}}$. They are described in the general case by the Lagrangian

$$
\begin{aligned}
\mathcal{L}_{\text {soft }}= & -\frac{1}{2} M_{g 3} \overline{\tilde{G}}^{(a) c} \tilde{G}^{(a)}-\frac{1}{2} M_{g 2} \overline{\tilde{W}}_{i}^{c} \tilde{W}_{i}-\frac{1}{2} M_{g 1} \overline{\tilde{B}}^{c} \tilde{B} \\
& -\left(M_{L}^{2}\right)^{i j} \tilde{l}_{L i}^{*} \tilde{l}_{L j}-\left(M_{E}^{2}\right)^{i j} \tilde{e}_{R i}^{*} \tilde{e}_{R j}-\left(\left(A_{E}\right)^{i j} \tilde{e}_{R i}^{*} \tilde{l}_{L j} \cdot H_{d}+\text { h.c }\right) \\
& -\left(m_{H 1}^{2}\left|H_{d}\right|^{2}-m_{H 2}^{2}\left|H_{u}\right|^{2}\right)-\left(B \mu H_{d} \cdot H_{u}+\text { h.c. }\right)-\cdots
\end{aligned}
$$

where $\tilde{G}^{(a)}, \tilde{W}_{i}$, and $\tilde{B}$ are the gauginos associated with the $\mathrm{SU}(3)_{c}, \mathrm{SU}(2)_{L}$, and $\mathrm{U}(1)_{Y}$ gauge symmetry groups, and $H_{u}, H_{d}, \tilde{l}_{L i}$, and $\tilde{e}_{R i}^{*}$ are the scalar components of $\hat{H}_{u}, \hat{H}_{d}, \hat{L}_{i}$, and $\hat{E}_{i}^{c}$ superfields, respectively, and the dots mean additional soft RH sneutrino and squark terms which do not play a role in our further discussion. We will consider in what follows the 
class of MSSMRN in which the soft SUSY breaking terms in eq. (16) are flavour-universal at the scale $M_{X}$,

$$
\begin{aligned}
& M_{g i}=m_{1 / 2}, \\
& m_{H 1}^{2}=m_{H 2}^{2}=m_{0}^{2}, \\
& \left(M_{L}\right)^{i j}=\left(M_{E}\right)^{i j}=m_{0}^{2} \delta^{i j}, \\
& \left(A_{E}\right)^{i j}=A_{0}\left(Y_{E}\right)^{i j}, \quad A_{0}=a_{0} m_{0}, \quad\left(Y_{E}\right)^{i j}=Y_{E}^{i} \delta^{i j} .
\end{aligned}
$$

In this case the only lepton flavour mixing present at $M_{X}$ is that due to the neutrino Yukawa couplings. However, at the scale $M_{R}=\min \left(M_{j}\right)=M_{1}$, where the RH neutrinos decouple, the RG running effects induce flavour non-diagonal elements in the effective soft SUSY breaking Lagrangian. The off-diagonal elements of interest for our further discussion are given in the leading-log approximation by [24],

$$
\begin{aligned}
& \left(M_{L}^{2}\right)^{i j} \simeq-\frac{1}{8 \pi^{2}} m_{0}^{2}\left(3+\left|a_{0}\right|^{2}\right) \sum_{k}\left(Y_{\nu}^{\dagger}\right)^{i k} \ln \frac{M_{X}}{M_{k}} Y_{\nu}^{k j}, \quad i \neq j, \\
& \left(M_{E}^{2}\right)^{i j} \simeq 0, \quad i \neq j, \\
& \left(A_{E}\right)^{i j} \simeq-\frac{3}{8 \pi^{2}} m_{0} a_{0} Y_{E}^{i} \sum_{k}\left(Y_{\nu}^{\dagger}\right)^{i k} \ln \frac{M_{X}}{M_{k}} Y_{\nu}^{k j}, \quad i \neq j .
\end{aligned}
$$

These terms generate new contributions in the amplitudes of the LFV processes. In the "mass insertion" (see, e.g., $[24,26,30]$ ) and leading-log approximations, the branching ratio of $l_{i} \rightarrow l_{j}+\gamma$ decay due to the new contributions has the following form

$$
\begin{aligned}
\operatorname{BR}\left(l_{i} \rightarrow l_{j} \gamma\right) & \simeq \frac{\Gamma\left(l_{i} \rightarrow e \nu \bar{\nu}\right)}{\Gamma_{\text {total }}\left(l_{i}\right)} \frac{\alpha_{\mathrm{em}}^{3}}{G_{F}^{2}} \frac{\left|\left(M_{L}^{2}\right)_{i j}\right|^{2}}{m_{S}^{8}} \tan ^{2} \beta \\
& \simeq \frac{\Gamma\left(l_{i} \rightarrow e \nu \bar{\nu}\right)}{\Gamma_{\text {total }}\left(l_{i}\right)} \frac{\alpha_{\mathrm{em}}^{3}}{G_{F}^{2} m_{S}^{8}}\left|\frac{\left(3+a_{0}^{2}\right) m_{0}^{2}}{8 \pi^{2}}\right|^{2}\left|\sum_{k}\left(Y_{\nu}^{\dagger}\right)^{i k} \ln \frac{M_{X}}{M_{k}} Y_{\nu}^{k j}\right|^{2} \tan ^{2} \beta
\end{aligned}
$$

where $m_{S}$ represents SUSY particle mass. It was shown in [30] that in most of the relevant soft SUSY breaking parameter space, the expression

$$
m_{S}^{8} \simeq 0.5 m_{0}^{2} m_{1 / 2}^{2}\left(m_{0}^{2}+0.6 m_{1 / 2}^{2}\right)^{2}
$$

gives an excellent approximation to the results obtained in a full renormalisation group analysis, i.e., without using the leading-log and the mass insertion approximations. Thus, in the case of soft SUSY breaking mediation by flavour-universal terms at $M_{X}>M_{R}$, the predicted rates of LFV processes such as $\mu \rightarrow e+\gamma$ decay are very sensitive to the off-diagonal elements of

$$
\mathbf{Y}_{\nu}^{\dagger}\left(M_{R}\right) \mathbf{Y}_{\nu}\left(M_{R}\right)=\frac{1}{v_{u}^{2}} \mathbf{U}\left(M_{R}\right) \sqrt{\mathbf{D}_{\nu}\left(M_{R}\right)} \mathbf{R}^{\dagger} \mathbf{D}_{N} \mathbf{R} \sqrt{\mathbf{D}_{\nu}\left(M_{R}\right)} \mathbf{U}^{\dagger}\left(M_{R}\right)
$$

Obviously, $\mathbf{Y}_{\nu}^{\dagger} \mathbf{Y}_{\nu}$ is affected by the RG running of $\mathbf{U}$ and $\mathbf{D}_{\nu}$ below the scale $M_{R}$, which is strongly dependent on $\tan \beta$, the light neutrino mass $m_{1}$ and the Majorana phase $\alpha$. 


\subsection{Results for $\mathrm{R}=1$}

In our further analysis we consider the case of quasi-degenerate heavy RH neutrinos, $M_{1}=M_{2}=M_{3}=M_{R}$ and we set $M_{X}=M_{\mathrm{GUT}}=2 \times 10^{16} \mathrm{GeV}$. We also first fix $\mathbf{R}$ in eq. (15) to be the unit matrix, $\mathbf{R}=\mathbf{1}$. The case of $\mathbf{R} \neq \mathbf{1}$ is discussed later. Under the conditions specified above the branching ratios $\mathrm{BR}\left(l_{i} \rightarrow l_{j}+\gamma\right)$ depend on $\mathbf{Y}_{\nu}^{\dagger} \mathbf{Y}_{\nu}$ which in turn takes the form

$$
\mathbf{Y}_{\nu}^{\dagger}\left(M_{R}\right) \mathbf{Y}_{\nu}\left(M_{R}\right)=\frac{M_{R}}{v_{u}^{2}} \mathbf{U}\left(M_{R}\right) \operatorname{diag}\left(m_{1}\left(M_{R}\right), m_{2}\left(M_{R}\right), m_{3}\left(M_{R}\right)\right) \mathbf{U}^{\dagger}\left(M_{R}\right)
$$

For the off-diagonal elements of $\mathbf{Y}_{\nu}^{\dagger} \mathbf{Y}_{\nu}$ of interest we get to leading order in small parameters

$$
\begin{aligned}
& \left(Y_{\nu}^{\dagger} Y_{\nu}\right)_{12} \cong \Delta_{21} c_{23} c_{12} s_{12} c_{13}+\Delta_{31} s_{23} c_{13} s_{13} e^{-i \delta}+\mathcal{O}\left(\Delta_{21} s_{13}\right) \\
& \left(Y_{\nu}^{\dagger} Y_{\nu}\right)_{13} \cong-\Delta_{21} s_{23} c_{12} s_{12} c_{13}+\Delta_{31} c_{23} c_{13} s_{13} e^{-i \delta}+\mathcal{O}\left(\Delta_{21} s_{13}\right) \\
& \left(Y_{\nu}^{\dagger} Y_{\nu}\right)_{23} \cong \Delta_{31} s_{23} c_{23} c_{13}^{2}+\mathcal{O}\left(\Delta_{21}, \Delta_{j i} s_{13}, s_{13}^{2}\right)
\end{aligned}
$$

where all angles should be evaluated at $M_{R}$ and

$$
\Delta_{i j} \equiv \frac{M_{R}}{v_{u}^{2}}\left(m_{i}\left(M_{R}\right)-m_{j}\left(M_{R}\right)\right)=\frac{M_{R}}{v_{u}^{2}} \frac{\Delta m_{i j}^{2}\left(M_{R}\right)}{m_{i}\left(M_{R}\right)+m_{j}\left(M_{R}\right)} .
$$

\subsubsection{The Case of Negligible RG Corrections}

The expressions for $\left(Y_{\nu}^{\dagger} Y_{\nu}\right)_{k l}, k \neq l=1,2,3$, we have derived, eqs. (25a)-(25c), are valid both for light neutrino mass spectrum with normal and inverted hierarchy, and, correspondingly, for $\mathrm{NH}, \mathrm{IH}$ and $\mathrm{QD}$ spectrum. If in the case of QD heavy $\mathrm{RH}$ neutrinos the RG effects on the angles $\theta_{i j}$ and masses $m_{j}$ are negligible or sufficiently small (e.g. if $\tan \beta \lesssim 10$ ), in the large region of the soft SUSY breaking parameter space where the approximate expression (21) with $m_{S}$ given by eq. (22) reproduces with a relatively high precision the values of $\mathrm{BR}\left(l_{i} \rightarrow l_{j}+\gamma\right)$ calculated exactly, the ratios

$$
\mathrm{R}(i j / k m) \equiv \frac{\mathrm{BR}\left(l_{i} \rightarrow l_{j}+\gamma\right)}{\operatorname{BR}\left(l_{k} \rightarrow l_{m}+\gamma\right)}, \quad i \neq j, k \neq m,
$$

depend only on the neutrino mixing parameters $\theta_{12}, \theta_{23}, \theta_{13}, \delta$ and $m_{j}$ at $M_{Z}$. We have, for instance, in the case of $\mathrm{NH}$ neutrino mass spectrum:

$$
\mathrm{R}(21 / 31) \equiv \frac{\mathrm{BR}(\mu \rightarrow e+\gamma)}{\operatorname{BR}(\tau \rightarrow e+\gamma)} \cong \frac{\left|\sqrt{\Delta m_{21}^{2}} c_{23} c_{12} s_{12}+\sqrt{\Delta m_{31}^{2}} s_{23} s_{13} e^{-i \delta}\right|^{2}}{\left|\sqrt{\Delta m_{21}^{2}} s_{23} c_{12} s_{12}-\sqrt{\Delta m_{31}^{2}} c_{23} s_{13} e^{-i \delta}\right|^{2}}, \mathrm{NH}
$$

The expressions for IH (QD) spectrum can obtained by replacing $\sqrt{\Delta m_{31}^{2}}$ with $\left(-2 \sqrt{\left|\Delta m_{31}^{2}\right|}\right)$ $\left(\sqrt{\Delta m_{21}^{2}}\right.$ and $\sqrt{\Delta m_{31}^{2}}$ with $\Delta m_{21}^{2}$ and $\left.\Delta m_{31}^{2}\right)$ in eq. (28). Depending on whether the terms $\propto \sqrt{\Delta m_{21}^{2}}$, or the terms $\propto \sqrt{\left|\Delta m_{31}^{2}\right|}$, dominate, we get independently of the type of neutrino mass spectrum

$$
\mathrm{R}(21 / 31) \cong \tan ^{-2} \theta_{23} \text { or } \tan ^{2} \theta_{23}
$$


In both cases $\operatorname{BR}(\mu \rightarrow e+\gamma)$ and $\operatorname{BR}(\tau \rightarrow e+\gamma)$ are predicted to differ at most by a factor $\sim 5$, which reflects the current $3 \sigma$ uncertainty in the value of $\tan ^{2} \theta_{23}$.

Similarly, we have for the ratio $\mathrm{R}(21 / 32)$ :

$$
\mathrm{R}(21 / 32) \equiv \frac{\mathrm{BR}(\mu \rightarrow e+\gamma)}{\operatorname{BR}(\tau \rightarrow \mu+\gamma)} \cong\left(\Delta m_{31}^{2}\right)^{-1}\left|\sqrt{\Delta m_{21}^{2}} c_{12} s_{12} s_{23}^{-1}+\sqrt{\Delta m_{31}^{2}} s_{13} c_{23}^{-1} e^{-i \delta}\right|^{2}, \mathrm{NH}
$$

The expression for IH (QD) spectrum can be obtained again by replacing $\sqrt{\Delta m_{31}^{2}}$ with $\left(-2 \sqrt{\left|\Delta m_{31}^{2}\right|}\right)\left(\sqrt{\Delta m_{21}^{2}}\right.$ and $\sqrt{\Delta m_{31}^{2}}$ with $\Delta m_{21}^{2}$ and $\left.\Delta m_{31}^{2}\right)$ in eq. (30). Now the dominance of the terms $\propto \sqrt{\Delta m_{21}^{2}}$ implies, e.g. for IH spectrum

$$
\mathrm{R}(21 / 32) \cong \frac{\Delta m_{21}^{2}}{4\left|\Delta m_{31}^{2}\right|} \frac{c_{12}^{2} s_{12}^{2}}{s_{23}^{2}}, \quad \mathrm{IH} .
$$

In the case of $\mathrm{NH}$ spectrum this ratio is 4 times bigger, while for QD spectrum the factor $\left(\Delta m_{21}^{2} /\left(4\left|\Delta m_{31}^{2}\right|\right)\right)$ should be replaced by $\left(\Delta m_{21}^{2} /\left|\Delta m_{31}^{2}\right|\right)^{2}$. If the terms $\propto \sqrt{\left|\Delta m_{31}^{2}\right|}$ dominate, we have independently of the type of the spectrum:

$$
\mathrm{R}(21 / 32) \cong s_{13}^{2} c_{23}^{-2} .
$$

In any of these cases we get $\mathrm{BR}(\mu \rightarrow e+\gamma) \ll \mathrm{BR}(\tau \rightarrow \mu+\gamma)$.

Let us add that for the current best fit values of the neutrino oscillation parameters and $\sin ^{2} \theta_{13}$ close to its current upper limit (7), the terms $\propto \sqrt{\Delta m_{21}^{2}}$ and $\propto \sqrt{\left|\Delta m_{31}^{2}\right|}$ in $\mathrm{R}(21 / 31)$ and $\mathrm{R}(21 / 32)$ are of the same order of magnitude for $\mathrm{NH}$ and $\mathrm{IH}$ neutrino mass spectra, while the term $\propto\left|\Delta m_{31}^{2}\right|$ is the dominant one for QD spectrum. Thus, if $\sin ^{2} \theta_{13}$ is sufficiently large and the neutrino mass spectrum is of the $\mathrm{NH}$ or $\mathrm{IH}$ type, the ratios $\mathrm{R}(21 / 31)$ and $\mathrm{R}(21 / 32)$ will depend on the Dirac CP-violation phase as well.

\subsubsection{RG Effects}

We turn next to the discussion of the RG effects. In order to find the changes due to the RG running from $M_{Z}$ to $M_{R}$ of the neutrino mass matrix and correspondingly of the neutrino mixing angles $\theta_{12}, \theta_{23}, \theta_{13}$ and masses $m_{j}$, the 1-loop RG equations in MSSMRN for the matrix $K_{N}$ in eq.(10), as we have already discussed, are solved numerically. As in the previous Section, the neutrino mass spectrum at $M_{Z}$ is assumed to be with normal hierarchy, $m_{1}\left(M_{Z}\right)<m_{2}\left(M_{Z}\right)<m_{3}\left(M_{Z}\right)$. The heavy RH neutrino mass scale $M_{R}$ is set at $M_{R}=2 \times 10^{13} \mathrm{GeV}$. The same best fit values of the neutrino mixing parameters at $M_{Z}$ were used as input in the calculation of the $R G$ running effects. In particular, we have fixed $\sin \theta_{13}=0$ at $M_{Z}$. The values of the neutrino mixing parameters at the scale $M_{R}$ were used as input in the calculation of the rates of the LFV decays $l_{i} \rightarrow l_{j}+\gamma$, which were performed for values of $\tan \beta$ from the interval $\sim(10-50)$ and a number of typical sets of values of the soft SUSY breaking parameters $m_{0}, m_{1 / 2}$ and $A_{0}$ in the few $100 \mathrm{GeV}$ range. These calculations have been performed without employing the reading-log and the mass insertion approximations.

The results we have obtained using the methods described above show that if $\mathbf{R}=\mathbf{1}$, taking into account the effects of the RG running of the neutrino mixing parameters in 
the case of relatively large $\tan \beta$ and/or $m_{1}$, can change significantly the predictions for the $\mathrm{LFV} l_{i} \rightarrow l_{j}+\gamma$ decay rates and the corresponding branching ratios $\operatorname{BR}\left(l_{i} \rightarrow l_{j}+\gamma\right)$. The magnitude of this change depends strongly on the value of the Majorana CP-violation phase $\alpha$. These conclusions are illustrated in Fig. 3, where the predicted $l_{i} \rightarrow l_{j}+\gamma$ decay branching ratios in the case of $\mathbf{R}=\mathbf{1}$ are shown as functions of the light neutrino mass $m_{1}$ for two typical sets of soft SUSY breaking parameters and three values of the Majorana CP-violation phase $\alpha, \alpha=0 ; \pi / 2 ; \pi$.

We find, in particular, that for $0.05 \lesssim m_{1} \lesssim 0.30 \mathrm{eV}$ and $30 \lesssim \tan \beta \lesssim 50$, the predicted $\mu \rightarrow e+\gamma$ and and $\tau \rightarrow e+\gamma$ decay rates, or $\operatorname{BR}(\mu \rightarrow e+\gamma)$ and $\operatorname{BR}(\tau \rightarrow e+\gamma)$, can be enhanced by the effects of the RG running of $\theta_{i j}$ and $m_{j}$ by 1 to 3 orders of magnitude if $\pi / 4 \lesssim \alpha \lesssim \pi$, while $\operatorname{BR}(\tau \rightarrow \mu+\gamma)$ can be enhanced by up to a factor of 10 . The enhancement of $\operatorname{BR}(\mu \rightarrow e+\gamma)$ and $\operatorname{BR}(\tau \rightarrow e+\gamma)$ is due to the "regeneration" of the terms $\propto \Delta_{31} s_{13}$ in $\left(Y_{\nu}^{\dagger} Y_{\nu}\right)_{12}$ and $\left(Y_{\nu}^{\dagger} Y_{\nu}\right)_{13}$ at $M_{R}$ : these terms are zero at $M_{Z}$ since we have set $s_{13}\left(M_{Z}\right)=0$. The corresponding enhancement factor in the case of $\mu \rightarrow e+\gamma$ and $\tau \rightarrow e+\gamma$ decays is given approximately by the ratio

$$
F \cong\left(\frac{\Delta m_{31}^{2}}{\Delta m_{21}^{2}} \frac{m_{1}+m_{2}}{m_{1}+m_{3}} \frac{s_{13}\left(M_{R}\right)}{c_{12} s_{12}}\right)^{2},
$$

where all quantities, and not only $s_{13}$, should be evaluated at $M_{R}$. For $\alpha=\pi$, however, $\theta_{12}\left(M_{R}\right) \cong \theta_{12}\left(M_{Z}\right)$ (see Fig. 11) and $F$ is reproduced with a rather good approximation if one uses the values of $\Delta m_{31}^{2}, \Delta m_{21}^{2}$ and of $m_{j}$ at $M_{Z}$. For $m_{1}=0.05(0.10) \mathrm{eV}$, for instance, we have for $\tan \beta=50, s_{13}\left(M_{R}\right) \cong 0.05$ (0.12) (see Fig. 1) and for the enhancement factor we find $F \cong 7$ (60). The case of $\tau \rightarrow e+\gamma$ decay is somewhat more complicated because of the possibility of accidental mutual cancellation for $\alpha \cong \pi$ of the two terms in eq. (25b) at $M_{R}{ }^{6}$. This can lead to a rather strong suppression (by more than an order of magnitude) of $\operatorname{BR}(\tau \rightarrow e+\gamma)$ in rather narrow interval of values of $m_{1}$. The "accidental" suppression under discussion can take place only in a limited region of the soft SUSY breaking parameter space.

For $\alpha \cong 0$, the combined effect of the RG running of $\theta_{12}, \theta_{23} \theta_{13}$ and $m_{j}$ on $\operatorname{BR}\left(l_{i} \rightarrow l_{j}+\gamma\right)$ is relatively small even for $m_{1} \sim(0.2-0.3) \mathrm{eV}$ and $\tan \beta \sim 50$. This is a consequence of the fact that for $\alpha=0, s_{13}$ essentially does not "run" and $s_{13}\left(M_{R}\right) \cong s_{13}\left(M_{Z}\right)$, the effect of RG running of $c_{23}\left(s_{23}\right)$ is relatively small, while the effects of RG running of $\Delta_{21}$ compensates the effect of running of $c_{12} s_{12}$ (see also Figs. 11 and 2). In this case $\operatorname{BR}(\mu \rightarrow$ $e+\gamma)$ and $\operatorname{BR}(\tau \rightarrow e+\gamma)(\operatorname{BR}(\tau \rightarrow \mu+\gamma))$ scale as functions of $m_{1}$ approximately as $\sim\left[\Delta m_{21(31)}^{2} /\left(v_{u}\left(m_{1}+m_{2(3)}\right)\right]^{2}\right.$, where $m_{2(3)}=\left(m_{1}^{2}+\Delta m_{21(31)}^{2}\right)^{\frac{1}{2}}$ and the masses $m_{j}$ and $\Delta m_{21,31}^{2}$ can be taken at $M_{Z}$.

The effects of the RG running of the neutrino mixing parameters $\theta_{12}, \theta_{23}, \theta_{13}$ and $m_{j}$ are, in general, considerably smaller for $\tan \beta \sim 10$. They are practically negligible for $\operatorname{BR}(\tau \rightarrow \mu+\gamma)$. If $\pi / 2 \lesssim \alpha \lesssim \pi$, they can lead to an enhancement of $\operatorname{BR}(\mu \rightarrow e+\gamma)$ approximately by a factor of $2.5(20)$ for $m_{1}=0.10(0.30) \mathrm{eV}$ and are essentially negligible if $m_{1}<0.06 \mathrm{eV}$. In what regards the $\tau \rightarrow e+\gamma$ decay branching ratio, $\operatorname{BR}(\tau \rightarrow e+\gamma)$, also in this case it can be strongly suppressed for $\alpha \cong \pi$ due to the accidental cancellation discussed above. Such a suppression can be realised for relatively large $m_{1} \gtrsim 0.15 \mathrm{eV}$ only.

\footnotetext{
${ }^{6}$ Note that since we set $s_{13}=0$ at $M_{Z}$, such a cancellation can take place only due to the fact that the RG running can generate a non-zero and sufficiently large $s_{13}$ at $M_{R}$.
} 


\subsection{The Case of $\mathrm{R} \neq 1$}

We shall analyse next briefly the case of nontrivial matrix $\mathbf{R}: \mathbf{R} \neq \mathbf{1}$. Successful leptogenesis can take place only if $\mathbf{R}$ is complex, so we will consider $(\mathbf{R})^{*} \neq \mathbf{R}$. The matrix $\mathbf{R}$ of interest, being a complex orthogonal matrix, can be written in the form: $\mathbf{R}=\mathbf{O} e^{i \mathbf{A}}$, where $\mathbf{O}$ is a real orthogonal matrix and $\mathbf{A}$ is a real antisymmetric matrix, $(\mathbf{A})^{T}=-\mathbf{A}$. In the case of QD heavy RH Majorana neutrinos we can set $\mathbf{O}=\mathbf{1}$ without loss of generality. Thus, in the subsequent calculations we use $\mathbf{R}=e^{i \mathbf{A}}$, parametrising the real antisymmetric matrix $\mathbf{A}$ as follows

$$
A=\left(\begin{array}{ccc}
0 & a & b \\
-a & 0 & c \\
-b & -c & 0
\end{array}\right)
$$

where $a, b, c$ are real. The following representation of $e^{i \mathbf{A}}$ proves convenient for our analysis $[28]$ :

$$
\mathbf{R}=e^{i \mathbf{A}}=\mathbf{1}-\frac{\cosh r-1}{r^{2}} \mathbf{A}^{2}+i \frac{\sinh r}{r} \mathbf{A},
$$

with $r=\sqrt{a^{2}+b^{2}+c^{2}}$. The requirement of successful leptogenesis in the case of QD light and heavy RH neutrino mass spectra implies [28] that $a b c \neq 0$ and that $10^{-5} \lesssim|a b c| \ll 1$. In what follows we will consider the range of values of the parameters $a, b, c$ determined by $|a|,|b|,|c| \lesssim 0.10$.

The matrix of neutrino Yukawa couplings in the case under discussion has the form $\mathbf{Y}_{\nu}=\frac{\sqrt{M_{R}}}{v_{u}} e^{i \mathbf{A}} \sqrt{\mathbf{D}_{\nu}} \mathbf{U}^{\dagger}$. The off-diagonal elements of $\mathbf{Y}_{\nu}^{\dagger} \mathbf{Y}_{\nu}$ of interest are given to leading order in the small quantities by

$$
\begin{aligned}
& \left(Y_{\nu}^{\dagger} Y_{\nu}\right)_{12}=\Delta_{21} c_{23} c_{12} s_{12} c_{13}+\Delta_{31} s_{23} c_{13} s_{13} e^{-i \delta} \\
& +2 \frac{M_{R}}{v_{u}^{2}} i\left[a \sqrt{m_{1} m_{2}}\left(c_{23}\left(c_{12}^{2} e^{-i \frac{\alpha}{2}}+s_{12}^{2} e^{i \frac{\alpha}{2}}\right)+s_{13} c_{12} s_{12} s_{23}\left(e^{i\left(\frac{\alpha}{2}-\delta\right)}-e^{-i\left(\frac{\alpha}{2}+\delta\right)}\right)\right)\right. \\
& +b \sqrt{m_{1} m_{3}} s_{23}\left(c_{12} e^{-i \frac{\beta_{M}}{2}}-s_{13} s_{12} e^{i\left(\frac{\beta_{M}}{2}-\delta\right)}\right) \\
& \left.+c \sqrt{m_{2} m_{3}} s_{23}\left(s_{12} e^{i \frac{\alpha-\beta_{M}}{2}}+s_{13} c_{12} e^{-i\left(\frac{\alpha-\beta_{M}}{2}+\delta\right)}\right)+\mathcal{O}\left(s_{13}^{2}\right)\right]+\mathcal{O}\left(r^{2}\right) \\
& \left(Y_{\nu}^{\dagger} Y_{\nu}\right)_{13}=-\Delta_{21} s_{23} c_{12} s_{12} c_{13}+\Delta_{31} c_{23} c_{13} s_{13} e^{-i \delta} \\
& +2 \frac{M_{R}}{v_{u}^{2}} i\left[a \sqrt{m_{1} m_{2}}\left(-s_{23}\left(c_{12}^{2} e^{-i \frac{\alpha}{2}}+s_{12}^{2} e^{i \frac{\alpha}{2}}\right)+s_{13} c_{12} s_{12} c_{23}\left(e^{i\left(\frac{\alpha}{2}-\delta\right)}-e^{-i\left(\frac{\alpha}{2}+\delta\right)}\right)\right)\right. \\
& \quad+b \sqrt{m_{1} m_{3}}\left(c_{12} c_{23} e^{-i \frac{\beta_{M}}{2}}-s_{13} s_{12} s_{23} e^{i\left(\frac{\beta_{M}}{2}-\delta\right)}\right) \\
& \left.\quad+c \sqrt{m_{2} m_{3}}\left(s_{12} c_{23} e^{i \frac{\alpha-\beta_{M}}{2}}+s_{13} c_{12} s_{23} e^{-i\left(\frac{\alpha-\beta_{M}}{2}+\delta\right)}\right)+\mathcal{O}\left(s_{13}^{2}\right)\right]+\mathcal{O}\left(r^{2}\right)
\end{aligned}
$$




$$
\begin{aligned}
&\left(Y_{\nu}^{\dagger} Y_{\nu}\right)_{23}=\Delta_{31} s_{23} c_{23} c_{13}^{2} \\
&+2 \frac{M_{R}}{v_{u}^{2}} i\left[a \sqrt { m _ { 1 } m _ { 2 } } \left(-2 i c_{12} s_{12} c_{23} s_{23} \sin \frac{\alpha}{2}+s_{13}\left[c_{12}^{2}\left(s_{23}^{2} e^{-i\left(\frac{\alpha}{2}-\delta\right)}+c_{23}^{2} e^{i\left(\frac{\alpha}{2}-\delta\right)}\right)\right.\right.\right. \\
&\left.\left.\quad+s_{12}^{2}\left(s_{23}^{2} e^{i\left(\frac{\alpha}{2}+\delta\right)}+c_{23}^{2} e^{-i\left(\frac{\alpha}{2}+\delta\right)}\right)\right]\right) \\
&+b \sqrt{m_{1} m_{3}}\left(-s_{12}\left(s_{23}^{2} e^{i \frac{\beta_{M}}{2}}+c_{23}^{2} e^{-i \frac{\beta_{M}}{2}}\right)+s_{13} c_{12} c_{23} s_{23} 2 i \sin \left(\frac{\beta_{M}}{2}-\delta\right)\right) \\
&+c \sqrt{m_{2} m_{3}}\left(c_{12}\left(c_{23}^{2} e^{i \frac{\alpha-\beta_{M}}{2}}+s_{23}^{2} e^{-i \frac{\alpha-\beta_{M}}{2}}\right)-s_{13} s_{12} c_{23} s_{23} 2 i \sin \left(\frac{\alpha-\beta_{M}}{2}+\delta\right)\right) \\
&+ \\
&\left.+\mathcal{O}\left(s_{13}^{2}\right)\right]+\mathcal{O}\left(r^{2}\right)
\end{aligned}
$$

Equations (36)-(38) are valid for any of the possible types of light neutrino mass spectrum. The above results imply that in the absence of significant RG effects, the "double" ratios $\mathrm{R}(21 / 31)=\mathrm{BR}(\mu \rightarrow e+\gamma) / \mathrm{BR}(\tau \rightarrow e+\gamma)$ and $\mathrm{R}(21 / 32)=\mathrm{BR}(\mu \rightarrow e+\gamma) / \mathrm{BR}(\tau \rightarrow$ $\mu+\gamma)$, depend in the region of validity of eqs. (21) and (22) in the relevant SUSY parameter space, not only on $\theta_{i j}, \delta$ and $m_{j}$ at $M_{Z}$, but also on the Majorana CP-violation phases $\alpha$ and $\beta_{M}$ and on the "leptogenesis parameters" $a, b$ and $c$.

The results we find for $\mathrm{BR}\left(l_{i} \rightarrow l_{j}+\gamma\right)$ taking into account the RG effects in the case of complex $\mathbf{R}$ given by eq. (35), are illustrated in Figs. 4 and $\mathbf{5}$, Both figures correspond to $\beta_{M}=0, \alpha=0 ; \pi / 2 ; \pi$, and the values of $\theta_{12}, \theta_{23}, \theta_{13}, \Delta m_{21}^{2}$ and $\Delta m_{31}^{2}$ at $M_{Z}$ used in Figs. 1. 3. In Fig. 4, the branching ratios $\operatorname{BR}(\mu \rightarrow e+\gamma), \operatorname{BR}(\tau \rightarrow e+\gamma)$, and $\operatorname{BR}(\tau \rightarrow \mu+\gamma)$ are shown as functions of $r \equiv \sqrt{a^{2}+b^{2}+c^{2}}$ varying in the interval $10^{-4}-5 \times 10^{-2}$ for fixed $m_{1}=0.06 \mathrm{eV}$ and SUSY parameters $\tan \beta=10, m_{1 / 2}=250 \mathrm{GeV}, m_{0}=100 \mathrm{GeV}$ and $A_{0}=-100 \mathrm{GeV}$. Figure $[5$ is similar to Fig. [3] but shows results for complex $\mathbf{R}$. It is obtained assuming that the three constants $a, b, c$ parametrising the matrix $\mathbf{R}$ lie in the interval $-0.1 \leq a, b, c \leq 0.1$. For comparison, in Fig. 5 results for $\mathbf{R}=\mathbf{1}$ are also shown ${ }^{7}$.

In the case of $\tan \beta \sim 10$ and $m_{1} \sim 0.06 \mathrm{eV}$ (Fig. 4), the RG effects are negligible. For $\alpha=0$, the branching ratios $\mathrm{BR}(\mu \rightarrow e+\gamma)$ and $\mathrm{BR}(\tau \rightarrow e+\gamma)$ can be significantly enhanced by the effect of complex $\mathbf{R}$ [28] for $r \gtrsim 5 \times 10^{-3}$ : the enhancement factor can be as large as $\sim 100$ at $r \cong 5 \times 10^{-2}$. Similar enhancement can take place for values of $\alpha$ in the interval $[\pi / 2, \pi]$. However, as Fig. 4 shows, for these relatively large values of $\alpha, B R(\mu \rightarrow e+\gamma)$ and $B R(\tau \rightarrow e+\gamma)$ can also be suppressed as a consequence of a partial cancellation between the contributions due to the complex $\mathbf{R}(r \neq 0)$ and the contribution present for $\mathbf{R}=\mathbf{1}$ (see eqs. (36)-(38) ). For the chosen values $m_{1}$ and $\beta_{M}$, the effect of complex $\mathbf{R}$ on $\operatorname{BR}(\tau \rightarrow \mu+\gamma)$ is minor: the latter can change at most by a factor $\sim 2$ for $r \lesssim 5 \times 10^{-2}$.

In the case of $|a|,|b|,|c| \leq 0.10$ and $\tan \beta=10$; 50, illustrated in Fig. 5 , $r$ can take somewhat larger values. For $\tan \beta=10$, the contributions due to the matrix $\mathbf{R}$ in $\operatorname{BR}(\mu \rightarrow$ $e+\gamma)$ and $\operatorname{BR}(\tau \rightarrow e+\gamma)$ become dominant at $m_{1} \gtrsim 0.01 \mathrm{eV}$; for $\operatorname{BR}(\tau \rightarrow \mu+\gamma)$ the same is valid at $m_{1} \gtrsim 0.10 \mathrm{eV}$. Correspondingly, for $m_{1} \gtrsim 0.01 \mathrm{eV}\left(m_{1} \gtrsim 0.10 \mathrm{eV}\right), \mathrm{BR}(\mu \rightarrow e+\gamma)$ and $\operatorname{BR}(\tau \rightarrow e+\gamma)(\operatorname{BR}(\tau \rightarrow \mu+\gamma))$ can be enhanced significantly independently of the value

\footnotetext{
${ }^{7}$ We should note that some of the predicted values of the branching ratio $\mathrm{BR}(\mu \rightarrow e \gamma)$, presented in Figs. [3 and 5 are already ruled out by the existing experimental limits on $\operatorname{BR}(\mu \rightarrow e \gamma)$. This illustrates, in particular, the type of constraints one might obtain, e.g. on the Majorana phase $\alpha$ and/or $m_{1}$ for a given specific set of values of SUSY breaking parameters.
} 
of the Majorana phase $\alpha$ (Fig. [5). For relatively large values of $\alpha$, e.g. $\alpha \sim[\pi / 2, \pi]$, there is again the possibility of a reduction (or suppression) of the $l_{i} \rightarrow l_{j}+\gamma$ decay rates of interest with respect to those predicted for $\mathbf{R}=1$ : the presence of the phase $\alpha$ can lead to partial cancellations between the three new terms proportional to $\sqrt{m_{1} m_{2}}, \sqrt{m_{1} m_{3}}$ and $\sqrt{m_{2} m_{3}}$ in eqs. (36)-(38). Similar conclusions are valid in the case of $\tan \beta=50$. For $\pi / 2 \lesssim \alpha \leq \pi$ in this case, the enhancement of $\mathrm{BR}(\mu \rightarrow e+\gamma)$ and $\mathrm{BR}(\tau \rightarrow e+\gamma)$ at $m_{1} \gtrsim 0.02 \mathrm{eV}$ due to the $R G$ effects is so strong that the contributions due to the complex $\mathbf{R}$ can lead to further increase of $\operatorname{BR}(\mu \rightarrow e+\gamma)$ and $\operatorname{BR}(\tau \rightarrow e+\gamma)$ by not more than approximately an order of magnitude. For sufficiently large $\alpha$, e.g. $\alpha \sim[\pi / 2, \pi]$, these contributions can also compensate partially the RG effect of enhancement leading to significantly smaller $\operatorname{BR}(\mu \rightarrow e+\gamma)$ and $\operatorname{BR}(\tau \rightarrow e+\gamma)$ than in the case of $\mathbf{R}=\mathbf{1}$ (Fig. 㧫. Qualitatively similar results are valid also for $\operatorname{BR}(\tau \rightarrow \mu+\gamma)$.

In the preceding discussion we have focused on the role of the Majorana phase $\alpha$, ignoring the possible effects of the second Majorana phase in the PMNS matrix $\beta_{M}$. Figures 4 and 5 correspond to $\beta_{M}=0$. It follows from eqs. (36)-(38) that if the phase $\beta_{M} \neq 0$ is sufficiently large, it can have an effect on the contributions to $\operatorname{BR}\left(l_{i} \rightarrow l_{j}+\gamma\right)$ due to the complex matrix $\mathbf{R}$, eq. (35), similar to that of the phase $\alpha$ discussed above.

\section{Conclusions}

In the present article we have investigate the effects of the Majorana CP-violation phases in the PMNS matrix and of the RG evolution of the light neutrino mixing parameters - masses and PMNS mixing angles, on the predictions for the rates of lepton flavour violating (LFV) decays, $\mu \rightarrow e+\gamma, \tau \rightarrow e+\gamma$ and $\tau \rightarrow \mu+\gamma$, in a class of SUSY theories with seesaw mechanism of neutrino mass generation. We have considered minimal supersymmetric extensions of the Standard Theory with heavy RH Majorana neutrinos (MSSMRN), in which soft SUSY breaking by flavour-universal terms in the Lagrangian occurs at a scale $M_{\mathrm{GUT}}$ above the RH Majorana neutrino mass scale $M_{R}$. In this class of theories, the charged lepton radiative decays $\mu \rightarrow e+\gamma, \tau \rightarrow \mu+\gamma$, etc. and other LFV processes are predicted to take place with rates that typically are within the reach of present and planned experiments. The predictions for the $\mu \rightarrow e+\gamma, \tau \rightarrow \mu+\gamma$, etc. decay rates are known to depend critically on the matrix of neutrino Yukawa couplings at the scale $M_{R}, \mathbf{Y}_{\nu}\left(M_{R}\right)$. The matrix $\mathbf{Y}_{\nu}\left(M_{R}\right)$ can be expressed in terms of the light and heavy RH Majorana neutrino masses, the PMNS mixing matrix $U-$ all at the scale $M_{R}$, and an orthogonal matrix $\mathbf{R}$. The same matrix $\mathbf{Y}_{\nu}\left(M_{R}\right)$ is one of the basic ingredients of the see-saw mechanism and of the leptogenesis which can take place only if the orthogonal matrix $\mathbf{R}$ is complex.

The neutrino mixing parameters entering into the expression for $\mathbf{Y}_{\nu}\left(M_{R}\right)$ should be evaluated at the scale $M_{R}$. These include the solar and atmospheric neutrino mixing angles $\theta_{12}$ and $\theta_{23}$, the CHOOZ angle $\theta_{13}$, the two Majorana CP-violating phases in the PMNS

matrix $\alpha$ and $\beta_{M}$ (see eq. (4) ), and the light neutrino masses $m_{1}, m_{2}=\sqrt{m_{1}^{2}+\Delta m_{21}^{2}}$, and $m_{3}=\sqrt{m_{1}^{2}+\Delta m_{31}^{2}}$, where $\Delta m_{21}^{2}$ and $\Delta m_{31}^{2}$ are the neutrino mass squared differences driving the solar and atmospheric neutrino oscillations. In order to find the changes of the mixing angles $\theta_{i j}$ and masses $m_{j}$, due to the RG evolution from the scale $M_{Z} \sim 100$ $\mathrm{GeV}$, where they are measured, to $M_{R}$, we have solved numerically the 1-loop RG equations 
in MSSMRN for the see-saw Majorana mass matrix of the left-handed flavour neutrinos, eq. (12). The light neutrino mass spectrum was assumed to be with normal hierarchy, $m_{1}<m_{2}<m_{3}$, and the lightest neutrino mass $m_{1}$ was allowed to take values from 0 to 0.30 $\mathrm{eV}$. The current best fit values of the known neutrino mixing angles and $\Delta m_{21(31)}^{2}$ at $M_{Z}$ (see eqs. (5) and (6) ) were used as initial conditions for the solution of the RG equations. We have presented results in the case of the CHOOZ mixing angle $\theta_{13}$ and the Majorana phase $\beta_{M}$ set to 0 at $M_{Z}$. In these and the subsequent calculations the scale $M_{R}$ was fixed at $M_{R}=2 \times 10^{13} \mathrm{GeV}$. Our results concerning the RG evolution of the neutrino mixing angles and neutrino mass differences $\left(m_{2(3)}-m_{1}\right)$ and $\Delta m_{21(31)}^{2}$ are discussed in Section 4 and are illustrated in Figs. 1 and 2, where the indicated quantities are shown at the scale $M_{R}$ as functions of $m_{1}$.

The values of the neutrino mixing parameters at the scale $M_{R}$ were used as input in the calculation of $\mathbf{Y}_{\nu}\left(M_{R}\right)$ and correspondingly of the rates of the LFV decays $l_{i} \rightarrow l_{j}+\gamma$. The latter were performed for a number of typical sets of values of the soft SUSY breaking parameters $\left(m_{0}, m_{1 / 2}, A_{0}\right)$ in the few $100 \mathrm{GeV}$ range and for values of $\tan \beta$ from the interval (10-50). For simplicity, the analyses were performed for the case of quasi-degenerate in mass heavy RH Majorana neutrinos, $M_{1}=M_{2}=M_{3}=M_{R}$. The rates of the LFV decays $\mu \rightarrow e+\gamma, \tau \rightarrow e+\gamma$ and $\tau \rightarrow \mu+\gamma$ have been calculated both in the cases of $\mathbf{R}=\mathbf{1}$ and of complex matrix $\mathbf{R}$.

Our results show that if $\mathbf{R}=\mathbf{1}$, taking into account the effects of the $\mathrm{RG}$ running of the neutrino mixing parameters in the case of relatively large $\tan \beta$ and/or $m_{1}$, can change significantly the predictions for the $l_{i} \rightarrow l_{j}+\gamma$ decay branching ratios $\operatorname{BR}\left(l_{i} \rightarrow l_{j}+\gamma\right)$. The magnitude of this change depends strongly on the value of the Majorana CP-violation phase $\alpha$. More specifically, we find that for $0.05 \lesssim m_{1} \lesssim 0.30 \mathrm{eV}$ and $30 \lesssim \tan \beta \lesssim 50$, the predicted branching ratios $\operatorname{BR}(\mu \rightarrow e+\gamma)$ and $\operatorname{BR}(\tau \rightarrow e+\gamma)$, can be enhanced by the effects of the RG running of $\theta_{i j}$ and $\left(m_{2(3)}-m_{1}\right)$ by 1 to 3 orders of magnitude if $\pi / 4 \lesssim \alpha \lesssim \pi$, while $\operatorname{BR}(\tau \rightarrow \mu+\gamma)$ can be enhanced by up to a factor of 10 . For $\alpha \cong 0$, the combined effect of the RG running of $\theta_{12}, \theta_{23} \theta_{13}$ and $m_{j}$ on $\mathrm{BR}\left(l_{i} \rightarrow l_{j}+\gamma\right)$ was found to be relatively small (essentially negligible) even for $m_{1} \sim(0.2-0.3) \mathrm{eV}$ and $\tan \beta \sim 50$.

We have considered further the case of non-trivial complex matrix $\mathbf{R}$ having the form given by eq. (35). In this case $\mathbf{R}$ can be parametrised by three real parameters $a, b, c$. The form of $\mathbf{R}$ we have employed is the most general relevant one for the study of the LFV decays $l_{i} \rightarrow l_{j}+\gamma$ in the case of QD heavy $\mathrm{RH}$ neutrinos. As it has been shown in [28], successful leptogenesis requires $a b c \neq 0$ and $10^{-5} \lesssim|a b c| \ll 1$. We find that in the case of $\tan \beta \sim 10$ and $m_{1} \sim 0.06 \mathrm{eV}$, i.e., when the RG effects are negligible, the branching ratios $\operatorname{BR}(\mu \rightarrow e+\gamma)$ and $\operatorname{BR}(\tau \rightarrow e+\gamma)$ can be significantly enhanced for any value of the Majorana phase $\alpha$ by the effect of complex $\mathbf{R}$ [28], provided $r \equiv \sqrt{a^{2}+b^{2}+c^{2}} \gtrsim 5 \times 10^{-3}$ (Fig. (4). The corresponding enhancement factor can be as large as $\sim 100$ for $r \cong 5 \times 10^{-2}$. For relatively large values of $\alpha$, e.g. $\alpha \sim[\pi / 2, \pi]$, however, and depending on the signs and values of the parameters $a, b, c, \operatorname{BR}(\mu \rightarrow e+\gamma)$ and $\operatorname{BR}(\tau \rightarrow e+\gamma)$ can also be suppressed as a consequence of a partial cancellation between the different contributions in the amplitudes of these two processes. The effect of complex $\mathbf{R}$ on $\operatorname{BR}(\tau \rightarrow \mu+\gamma)$ is minor for $r \lesssim 5 \times 10^{-2}$ and $m_{1}<0.10 \mathrm{eV}$. In the case of $a, b, c$ satisfying $|a|,|b|,|c| \leq 0.10$, the contributions due to $\mathbf{R}$ in $\operatorname{BR}(\mu \rightarrow e+\gamma)$ and $\operatorname{BR}(\tau \rightarrow e+\gamma)$ become dominant at $m_{1} \gtrsim 0.01 \mathrm{eV}$; for $\operatorname{BR}(\tau \rightarrow \mu+\gamma)$ they become dominant at $m_{1} \gtrsim 0.10 \mathrm{eV}$. Similar results are valid in the 
case of $\tan \beta \sim 50$. For $\pi / 2 \lesssim \alpha \leq \pi$ in this case, the enhancement of $\operatorname{BR}(\mu \rightarrow e+\gamma)$ and $\mathrm{BR}(\tau \rightarrow e+\gamma)$ at $m_{1} \gtrsim 0.02 \mathrm{eV}$ due to the RG effects is so strong that the contributions of the complex $\mathbf{R}$ can lead to further increase of $\operatorname{BR}(\mu \rightarrow e+\gamma)$ and $\operatorname{BR}(\tau \rightarrow e+\gamma)$ by not more than approximately an order of magnitude. For sufficiently large $\alpha$, e.g. $\alpha \sim[\pi / 2, \pi]$, these contributions can also compensate partially the $\mathrm{RG}$ effect of enhancement leading to

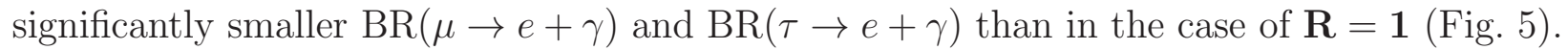
Qualitatively similar results are valid also for $\operatorname{BR}(\tau \rightarrow \mu+\gamma)$. If the Majorana phase $\beta_{M}$ is sufficiently large, it can have an effect on the contributions to $\mathrm{BR}\left(l_{i} \rightarrow l_{j}+\gamma\right)$ due to the complex matrix $\mathbf{R}$, similar to that of the phase $\alpha$ discussed above.

The results of the present study show that taking into account the effects of the Majorana CP-violation phases in the PMNS matrix and of the RG evolution of the light neutrino mixing parameters - masses and PMNS mixing angles, can have a remarkably large impact on the predicted rates of the LFV decays $\mu \rightarrow e+\gamma$ and $\tau \rightarrow e+\gamma$, etc. in MSSM with right-handed neutrinos and see-saw mechanism of neutrino mass generation. The impact of these effects on the $\tau \rightarrow \mu+\gamma$ decay rate is typically smaller and possible only for $m_{1} \gtrsim 0.10 \mathrm{eV}$.

Acknowledgements. S.T.P. would like to thank the Institute of Nuclear Theory at the University of Washington, Seattle, for its hospitality, and the U.S. Department of Energy for partial support, during the completion of the present study. This work was supported in part by the Italian INFN under the program "Fisica Astroparticellare" (S.T.P. and T.S.).

\section{APPENDIX A}

In the present Appendix we derive approximate analytic expressions for some of the neutrino mixing parameters at the scale $M_{R}$. The renormalisation group (RG) equation for the neutrino mass matrix $m_{\nu}$ in MSSMRN has the form (see, e.g., [37])

$$
\frac{d}{d \ln \mu_{R}} m_{\nu}=\frac{1}{8 \pi^{2}}\left[\left\{-4 \pi\left(3 \alpha_{2}+\frac{3}{5} \alpha_{1}\right)+\operatorname{tr}\left(3 Y_{U}^{\dagger} Y_{U}\right)\right\} m_{\nu}+\frac{1}{2}\left\{\left(Y_{E}^{\dagger} Y_{E}\right)^{T} m_{\nu}+m_{\nu}\left(Y_{E}^{\dagger} Y_{E}\right)\right\}\right]
$$

where $\mu_{R}$ is the RG energy scale, $\alpha_{j}=g_{j}^{2} /(4 \pi)$, where $g_{3}, g_{2}$ and $g_{1}$ are the $S U(3)^{c}, S U(2)_{L}$ and $U(1)_{Y}$ gauge coupling constants, respectively, and $Y_{U}$ is the matrix of Yukawa couplings of up-quarks. This equation admits the approximate solution [34]

$$
m_{\nu}\left(M_{R}\right) \simeq J\left(M_{R}\right)\left(\begin{array}{ccc}
1 & & \\
& 1 & \\
& & 1+\epsilon_{\tau}
\end{array}\right) m_{\nu}\left(M_{Z}\right)\left(\begin{array}{ccc}
1 & & \\
& 1 & \\
& & 1+\epsilon_{\tau}
\end{array}\right)
$$

where $J\left(M_{R}\right)$ and $\epsilon_{\tau}$ are given by

$$
\begin{aligned}
J\left(M_{R}\right) & =\exp \left[\frac{1}{8 \pi^{2}} \int_{\ln M_{Z}}^{\ln M_{R}}\left(\operatorname{tr}\left(3 Y_{U}^{\dagger}(\mu) Y_{U}(\mu)\right)-4 \pi\left(3 \alpha_{2}(\mu)+\frac{3}{5} \alpha_{1}(\mu)\right)\right) d(\ln \mu)\right], \\
\epsilon_{\tau} & =1-\left(\frac{M_{Z}}{M_{R}}\right)^{\left(1 / 8 \pi^{2}\right)\left(1+\tan ^{2} \beta\right)\left(m_{\tau} / v\right)^{2}},
\end{aligned}
$$


$m_{\tau}$ being the $\tau^{ \pm}$mass. Note that $\epsilon_{\tau}$ takes positive values and is a monotonically increasing function of $\tan \beta$. The PMNS matrix at $M_{R}, U\left(M_{R}\right)$, is obtained by diagonalising $m_{\nu}\left(M_{R}\right)$ :

$$
U\left(M_{R}\right)^{T} m_{\nu}\left(M_{R}\right) U\left(M_{R}\right)=\operatorname{diag}\left(m_{1}\left(M_{R}\right), m_{2}\left(M_{R}\right), m_{3}\left(M_{R}\right)\right) .
$$

Using eqs. (44) and (40) - (43) it is possible to derive the following approximate expressions for the solar neutrino mixing angle $\theta_{12}$ and $\Delta m_{21}^{2}$ at $M_{R}$ :

$$
\tan \theta_{12}\left(M_{R}\right) \simeq \frac{\left|\sin \theta_{12}\left(M_{Z}\right) \cos \theta_{x}+\cos \theta_{12}\left(M_{Z}\right) \sin \theta_{x} e^{-i \alpha / 2}\right|}{\left|\cos \theta_{12}\left(M_{Z}\right) \cos \theta_{x}-\sin \theta_{12}\left(M_{Z}\right) \sin \theta_{x} e^{-i \alpha / 2}\right|},
$$

and

$$
\Delta m_{21}^{2}\left(M_{R}\right) \simeq J^{2}\left(M_{R}\right) \frac{\Delta m_{21}^{2}\left(M_{Z}\right)+4 \epsilon_{\tau} m_{1}^{2}\left(M_{Z}\right) \sin ^{2} \theta_{23}\left(M_{Z}\right) \cos 2 \theta_{12}\left(M_{Z}\right)}{\cos 2 \theta_{x}}
$$

where the angle $\theta_{x}$ is determined by the equation

$$
\tan 2 \theta_{x} \simeq-\frac{4 \epsilon_{\tau} m_{1}^{2}\left(M_{Z}\right) \sin ^{2} \theta_{23}\left(M_{Z}\right) \sin 2 \theta_{12}\left(M_{Z}\right) \cos \frac{\alpha\left(M_{Z}\right)}{2}}{\Delta m_{21}^{2}\left(M_{Z}\right)+4 \epsilon_{\tau} m_{1}^{2}\left(M_{Z}\right) \sin ^{2} \theta_{23}\left(M_{Z}\right) \cos 2 \theta_{12}\left(M_{Z}\right)} .
$$

As can be shown, eqs. (45) and (46) imply $\cos 2 \theta_{x}>0, \sin 2 \theta_{x}<0$, provided we keep the ordering $m_{1}\left(M_{R}\right)<m_{2}\left(M_{R}\right)$ of the masses $m_{1}$ and $m_{2}$ at $M_{R}$. It follows, in particular, from eq. (44) that

$$
\sin ^{2} 2 \theta_{12}\left(M_{R}\right) \leq \sin ^{2} 2 \theta_{12}\left(M_{Z}\right)
$$

For $\alpha\left(M_{Z}\right)=0$ and $\alpha\left(M_{Z}\right)=\pi$, for instance, we have

$$
\sin ^{2} 2 \theta_{12}\left(M_{R}\right)= \begin{cases}\sin ^{2} 2\left(\theta_{12}\left(M_{Z}\right)+\theta_{x}\right), & \alpha=0 \\ \sin ^{2} 2 \theta_{12}\left(M_{Z}\right), & \alpha=\pi\end{cases}
$$

The mixing angle $\theta_{13}$ and the Dirac CP-violation phase $\delta$ are also affected by the RG evolution from $M_{Z}$ to $M_{R}$. If, e.g. we set $s_{13}=0$ at $M_{Z}$, we get for $s_{13}$ and $\delta$ at $M_{R}$ :

$$
s_{13}\left(M_{R}\right) \simeq \frac{J^{2}\left(M_{R}\right) \epsilon_{\tau} m_{1}\left(M_{Z}\right) m_{3}\left(M_{Z}\right) \sin 2 \theta_{12}\left(M_{Z}\right) \sin 2 \theta_{23}\left(M_{Z}\right)}{m_{3}^{2}\left(M_{R}\right)-m_{1}^{2}\left(M_{R}\right)} \sin \frac{\alpha\left(M_{Z}\right)}{2}
$$

and

$$
\delta\left(M_{R}\right) \simeq \xi_{1}+\xi_{2}-\frac{\pi}{2}+\frac{\alpha\left(M_{Z}\right)}{2}-\beta_{M}\left(M_{Z}\right), \quad \text { for } \alpha\left(M_{Z}\right) \neq 0
$$

where

$$
\begin{aligned}
& \xi_{1}=\arg \left(c_{12}\left(M_{Z}\right) c_{x}-s_{12}\left(M_{Z}\right) s_{x} e^{-i \alpha\left(M_{Z}\right) / 2}\right) \\
& \xi_{2}=\arg \left(s_{12}\left(M_{Z}\right) c_{x}+c_{12}\left(M_{Z}\right) s_{x} e^{i \alpha\left(M_{Z}\right) / 2}\right)
\end{aligned}
$$

The angle $\theta_{13}\left(M_{R}\right)$ takes its largest value for $\alpha\left(M_{Z}\right)=\pi$ and in this case $\delta\left(M_{R}\right) \cong-\beta_{M}\left(M_{Z}\right)$. In contrast, if $\alpha=0$, the correction to $\sin \theta_{13}$ due to the RG running is negligible. 


\section{References}

[1] B. T. Cleveland et al., Astrophys. J. 496 (1998) 505; Y. Fukuda et al. [Kamiokande Collaboration], Phys. Rev. Lett. 77 (1996) 1683; J. N. Abdurashitov et al. [SAGE Collaboration], J. Exp. Theor. Phys. 95 (2002) 181; T. Kirsten et al. [GALLEX and GNO Collaborations], Nucl. Phys. B (Proc. Suppl.) 118 (2003) 33; C. Cattadori et al., Nucl. Phys. B (Proc. Suppl.) 143 (2005) 3.

[2] S. Fukuda et al. [Super-Kamiokande Collaboration], Phys. Lett. B539 (2002) 179; Y. Fukuda et al. Phys. Rev. Lett. 81 (1998) 1562; Y. Ashie et al., Phys. Rev. Lett. 93 (2004) 101801.

[3] Q. R. Ahmad et al. [SNO Collaboration], Phys. Rev. Lett. 87 (2001) 071301; ibid. 89 (2002) 011301 and 011302; S. N. Ahmed et al., Phys. Rev. Lett. 92 (2004) 181301; B. Aharmim et al., nucl-ex/0502021.

[4] K. Eguchi et al. [KamLAND Collaboration], Phys. Rev. Lett. 90 (2003) 021802; T. Araki et al., hep-ex/0406035.

[5] E. Aliu et al. [K2K Collaboration], hep-ex/0411038

[6] S. T. Petcov, Nucl. Phys. B (Proc. Suppl.) 143 (2005) 159.

[7] B. Pontecorvo, Zh. Eksp. Teor. Fiz. (JETP) 33 (1957) 549; ibid. 34 (1958) 247; ibid. 53 (1967) 1717; Z. Maki, M. Nakagawa and S. Sakata, Prog. Theor. Phys. 28 (1962) 870.

[8] V. Lobashev et al., Nucl. Phys. A719(2003) 153c; K. Eitel et al., Nucl. Phys. B (Proc. Suppl.) 143 (2005) 197.

[9] D. N. Spergel et al. [WMAP Collaboration], Astrophys. J. Suppl. 148 (2003) 175.

[10] S. Hannestad, astro-ph/0303076 O. Elgaroy and O. Lahav, astro-ph/0303089.

[11] P. Minkowski, Phys. Lett. B67 (1977) 421; M. Gell-Mann, P. Ramond, and R. Slansky in Supergravity, p. 315, edited by F. Nieuwenhuizen and D. Friedman, North Holland, Amsterdam, 1979; T. Yanagida, Proc. of the Workshop on Unified Theories and the Baryon Number of the Universe, edited by O. Sawada and A. Sugamoto, KEK, Japan 1979; R. N. Mohapatra, G. Senjanović, Phys. Rev. Lett. 44 (1980) 912.

[12] B. Pontecorvo, Zh. Eksp. Teor. Fiz. 53 (1967) 1717; S. M. Bilenky and B. Pontecorvo, Lett. Nuov. Cim. 17 (1976) 569.

[13] M. Fukugita and T. Yanagida, Phys. Lett. B174 (1986) 45.

[14] S. M. Bilenky and S. T. Petcov, Rev. Mod. Phys. 59 (1987) 671.

[15] S. T. Petcov, Sov. J. Nucl. Phys. 25 (1977) 340.

[16] S. M. Bilenky, S. T. Petcov and B. Pontecorvo, Phys. Lett. B67 (1977) 309; T. P. Cheng and L.-F. Li, Phys. Rev. Lett. 45 (1980) 1908. 
[17] M. L. Brooks et al. [MEGA Collaboration], Phys. Rev. Lett. 83 (1999) 1521.

[18] S. Eidelman et al. [Particle Data Group], Phys. Lett. B592 (2004) 1.

[19] B. Aubert et al. [BABAR Collaboration], hep-ex/0502032.

[20] Y. Kuno and Y. Okada, Rev. Mod. Phys. 73 (2001) 151.

[21] L. M. Barkov et al., the MEG Proposal (1999), http://meg.psi.ch.

[22] F. Borzumati and A. Masiero, Phys. Rev. Lett. 57 (1986) 961.

[23] R. Barbieri, S. Ferrara and C. Savoy, Phys. Lett. B119 (1982) 343; L. Hall, J. Lykken and S. Weinberg, Phys. Rev. D27 (1983) 2359.

[24] J. Hisano et al., Phys. Lett. B357 (1995) 579; Phys. Rev. D53 (1996) 2442; J. Hisano and D. Nomura, Phys. Rev. D59 (1999) 116005.

[25] J. A. Casas and A. Ibarra, Nucl. Phys. B618 (2001) 171.

[26] J. Ellis et al., Nucl. Phys. B621 (2002) 208; Phys. Rev. D66 (2002) 115013; J. Ellis and M. Raidal, Nucl. Phys. B643 (2002) 229; J. Ellis, M. Raidal and T. Yanagida, Phys. Lett. B546 (2002) 228.

[27] S. Lavignac, I. Masina and C. A. Savoy, Phys. Lett. B520 (2001) 269; A. Kageyama et al., Phys. Rev. D65 (2002) 096010; Phys. Lett. B527 (2002) 206; F. Deppisch et al., Eur. Phys. J. C28 (2003) 365; T. Blazek and S. F. King, Nucl. Phys. B662 (2003) 359; B. Dutta and R. N. Mohapatra, Phys. Rev. D68 (2003) 056006; J. I. Illana and M. Masip, Eur. Phys. J. C35 (2004) 365; A. Masiero, S. K. Vempati and O. Vives, New J. Phys. 6 (2004) 202; M. Bando et al., hep-ph/0405071; I. Masina and C. A. Savoy, Phys. Rev. D71 (2005) 093003; K. S. Babu, J. C. Pati and P. Rastogi, hep-ph/0502152; P. Paradisi, hep-ph/0505046.

[28] S. Pascoli, S. T. Petcov and C. E. Yaguna, Phys. Lett. B564 (2003) 241.

[29] S. Pascoli, S. T. Petcov and W. Rodejohann, Phys. Rev. D68 (2003) 093007.

[30] S. T. Petcov et al., Nucl. Phys. B676 (2004) 453.

[31] S. Kanemura et al., hep-ph/0501228, hep-ph/0507264.

[32] S. M. Bilenky, J. Hosek and S. T. Petcov, Phys. Lett. B94 (1980) 495.

[33] P. H. Chankowski and Z. Płuciennik, Phys. Lett. B316 (1993) 312; K. S. Babu, C. N. Leung and J. Pantaleone, Phys. Lett. B319 (1993) 191; M. Tanimoto, Phys. Lett. B360 (1995) 41.

[34] J. Ellis and S. Lola, Phys. Lett. B458 (1999) 310; J. A. Casas et al., Nucl. Phys. B556 (1999) 3; ibid. B569 (2000) 822; N. Haba and N. Okamura, Eur. Phys. J. C14 (2000) 347; N. Haba et al., Prog. Theor. Phys. 103 (2000) 145. 
[35] J. A. Casas et al., Nucl. Phys. B573 (2000) 652; C. D. Froggatt, H. B. Nielsen and Y. Takanishi, Nucl. Phys. B631 (2002) 285.

[36] T. Miura, T. Shindou and E. Takasugi, Phys. Rev. D66 (2002) 093002; Nucl. Phys. A721 (2003) 537; R. N. Mohapatra, M. K. Parida and G. Rajasekaran, Phys. Rev. D69 (2004) 053007.

[37] S. Antusch et al., Nucl. Phys. B674 (2003) 401.

[38] S. M. Bilenky, S. Pascoli and S. T. Petcov, Phys. Rev. D64 (2001) 053010.

[39] J. Schechter and J. W. F. Valle, Phys. Rev. D22 (1980) 2227; M. Doi et al., Phys. Lett. B102 (1981) 323; J. Bernabeu and P. Pascual, Nucl. Phys. B228 (1983) 21.

[40] M. Apollonio et al., Phys. Lett. B466 (1999) 415; F. Boehm et al., Phys. Rev. Lett. 84 (2000) 3764.

[41] A. Bandyopadhyay et al., Phys. Lett. B608 (2005) 115.

[42] J. N. Bahcall, M. C. Gonzalez-Garcia and C. Peña-Garay, JHEP 0408 (2004) 016; M. Maltoni et al., New J. Phys. 6122 (2004) 122.

[43] A. Bandyopadhyay et al., Phys. Lett. B583 (2004) 134.

[44] S. Pascoli and S. T. Petcov, Phys. Lett. B544 (2002) 239; ibid. B580 (2004) 280.

[45] C. Aalseth et al., hep-ph/0412300 A. Morales and J. Morales, Nucl. Phys. B (Proc. Suppl.) 114 (2003) 141.

[46] P. Langacker et al., Nucl. Phys. B282 (1987) 589.

[47] S. T. Petcov, New J. Phys. 6 (2004) 109 (http://stacks.iop.org/1367-2630/6/109); Talk given at the Nobel Symposium (N 129) on Neutrino Physics, August 19 - 24, 2004, Haga Slot, Enköping, Sweden, hep-ph/0504110, S. Pascoli and S. T. Petcov, hep-ph/0308034.

[48] S. M. Bilenky et al., Phys. Rev. D56 (1996) 4432.

[49] S. Pascoli, S. T. Petcov and L. Wolfenstein, Phys. Lett. B524 (2002) 319; S. Pascoli, S. T. Petcov and W. Rodejohann, Phys. Lett. B549 (2002) 177; S. Pascoli, S. T. Petcov and T. Schwetz, hep-ph/0505226.

[50] L. Wolfenstein, Phys. Lett. B107 (1981) 77; S. M. Bilenky, N. P. Nedelcheva and S. T. Petcov, Nucl. Phys. B247 (1984) 61; B. Kayser, Phys. Rev. D30 (1984) 1023. 

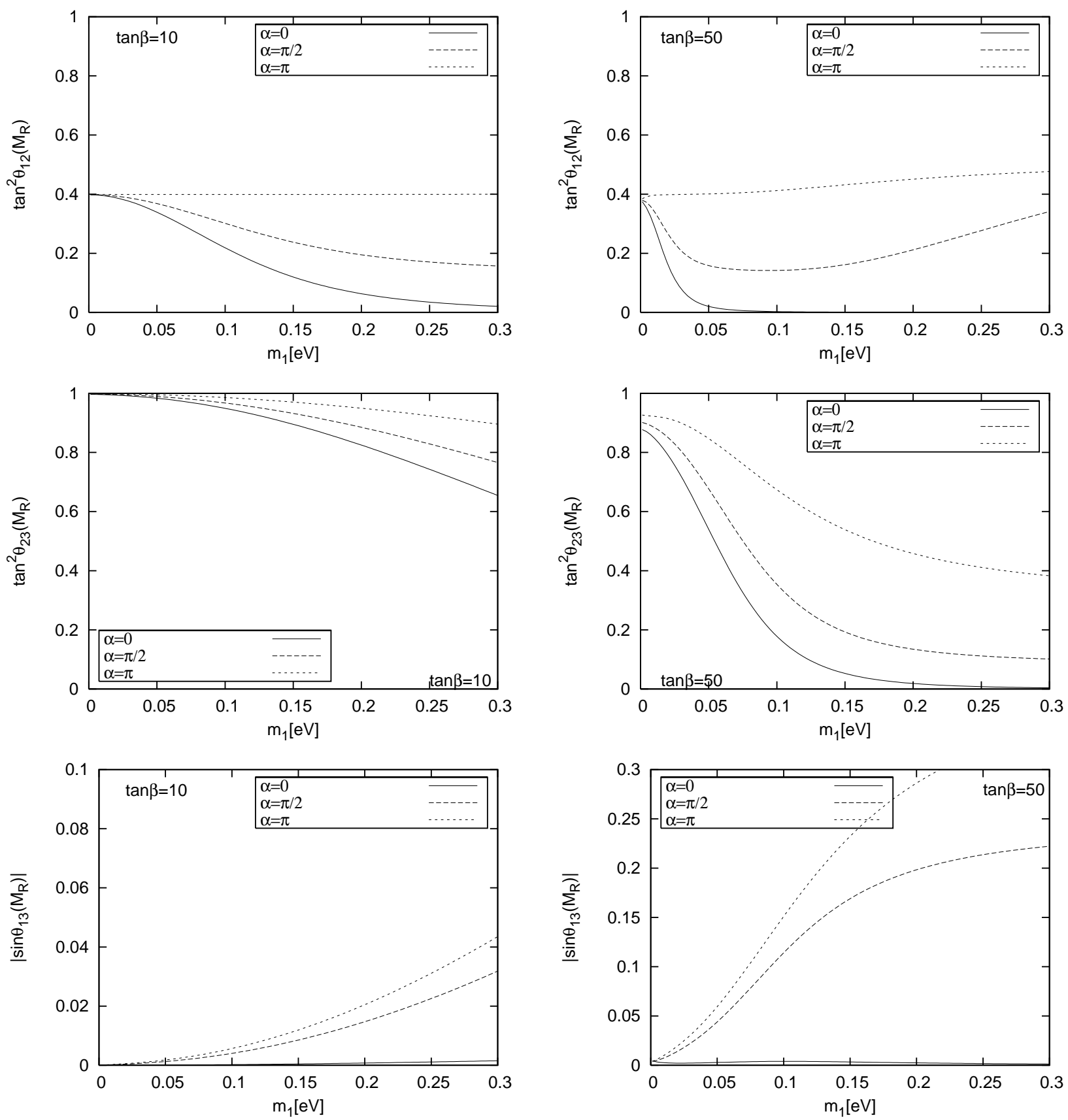

Figure 1: The dependence of $\tan ^{2} \theta_{\text {sol }} \equiv \tan ^{2} \theta_{12}, \tan ^{2} \theta_{\text {atm }} \equiv \tan ^{2} \theta_{23}$ and of $\left|V_{13}\right| \equiv \sin \theta_{13}$, evaluated at $M_{R}$, on the lightest neutrino mass $m_{1}$ for $\tan \beta=10$ and $\tan \beta=50$. The following values neutrino mixing parameters at $M_{Z}$ were used as input in the calculation of the RG running effects: $\Delta m_{\odot}^{2}=8.0 \times 10^{-5} \mathrm{eV}^{2}, \Delta m_{A}^{2}=2.2 \times 10^{-3} \mathrm{eV}^{2}, \tan ^{2} \theta_{\odot}=0.4$, $\tan ^{2} \theta_{A}=1$, and $\sin \theta_{13}=0.0$. The neutrino mass spectrum at $M_{Z}$ is assumed to be with normal hierarchy, $m_{1}\left(M_{Z}\right)<m_{2}\left(M_{Z}\right)<m_{3}\left(M_{Z}\right)$. The RH neutrino mass scale $M_{R}$ is fixed as $M_{R}=2 \times 10^{13} \mathrm{GeV}$. 


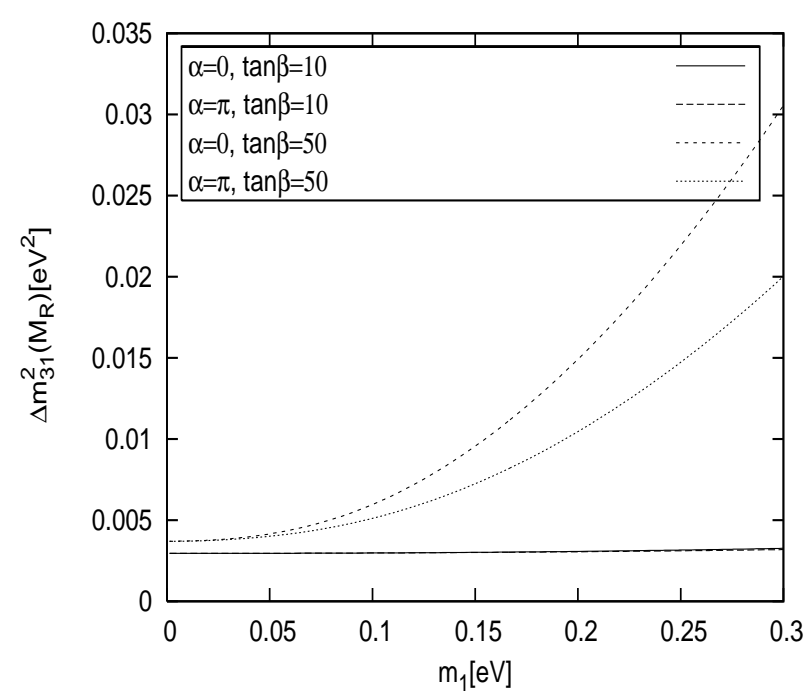

(a)

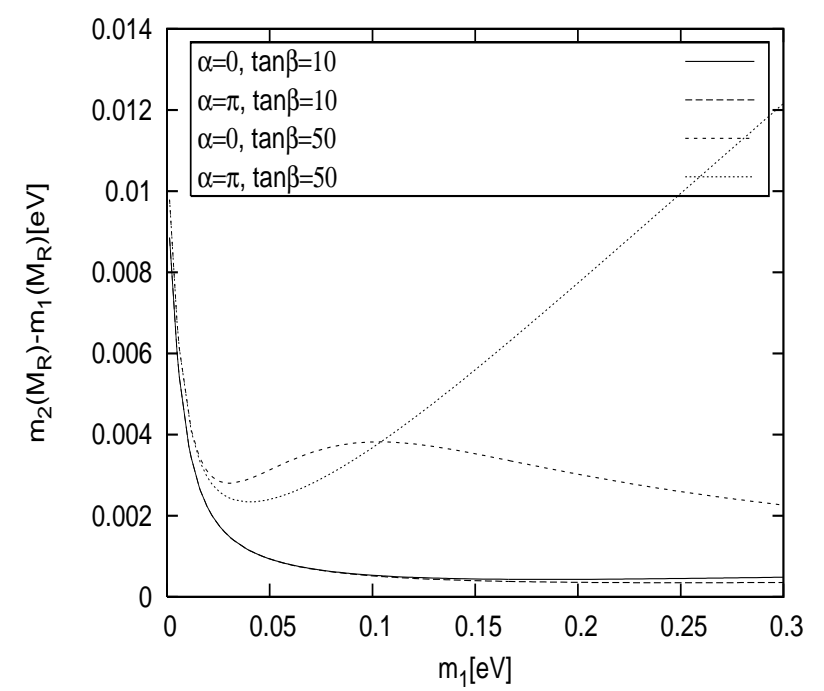

(c)

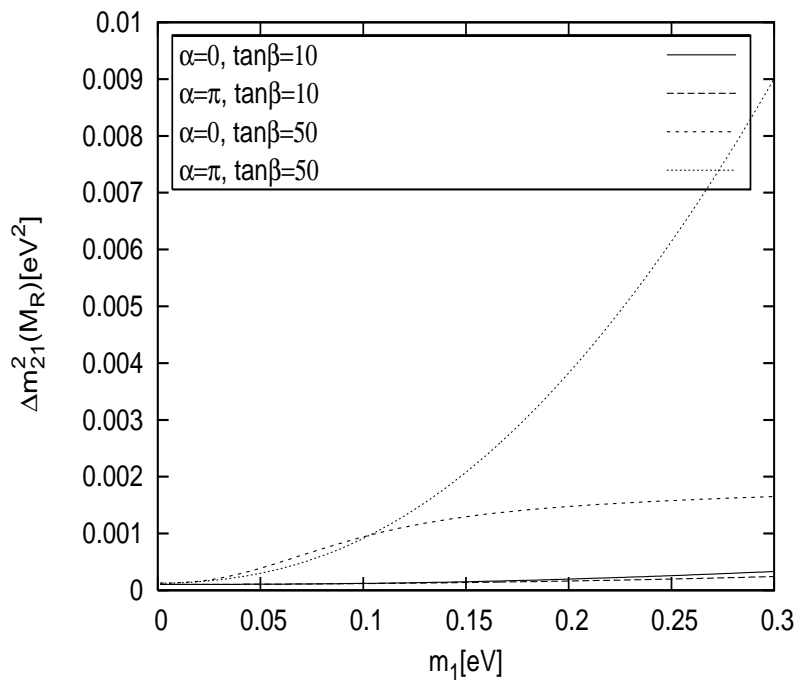

(b)

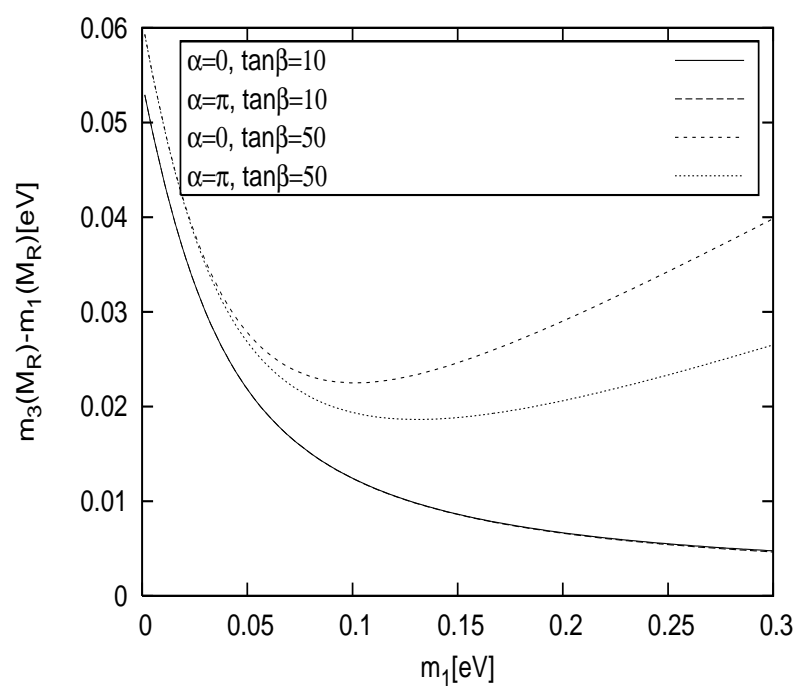

(d)

Figure 2: The light neutrino mass differences at the scale $M_{R}$ as a functions of $m_{1}$ for $\alpha=0 ; \pi$ and $\tan \beta=10 ; 50$ : (a) $\Delta m_{A}^{2}\left(M_{R}\right) \equiv m_{3}^{2}\left(M_{R}\right)-m_{1}^{2}\left(M_{R}\right)$, (b) $\Delta m_{\odot}^{2}\left(M_{R}\right) \equiv$ $m_{2}^{2}\left(M_{R}\right)-m_{1}^{2}\left(M_{R}\right)$, (c) $m_{2}\left(M_{R}\right)-m_{1}\left(M_{R}\right)$, and (d) $m_{3}\left(M_{R}\right)-m_{1}\left(M_{R}\right)$. The neutrino mixing parameters at $M_{Z}$ and the mass $M_{R}$ used are the same as in Fig. 1 . 

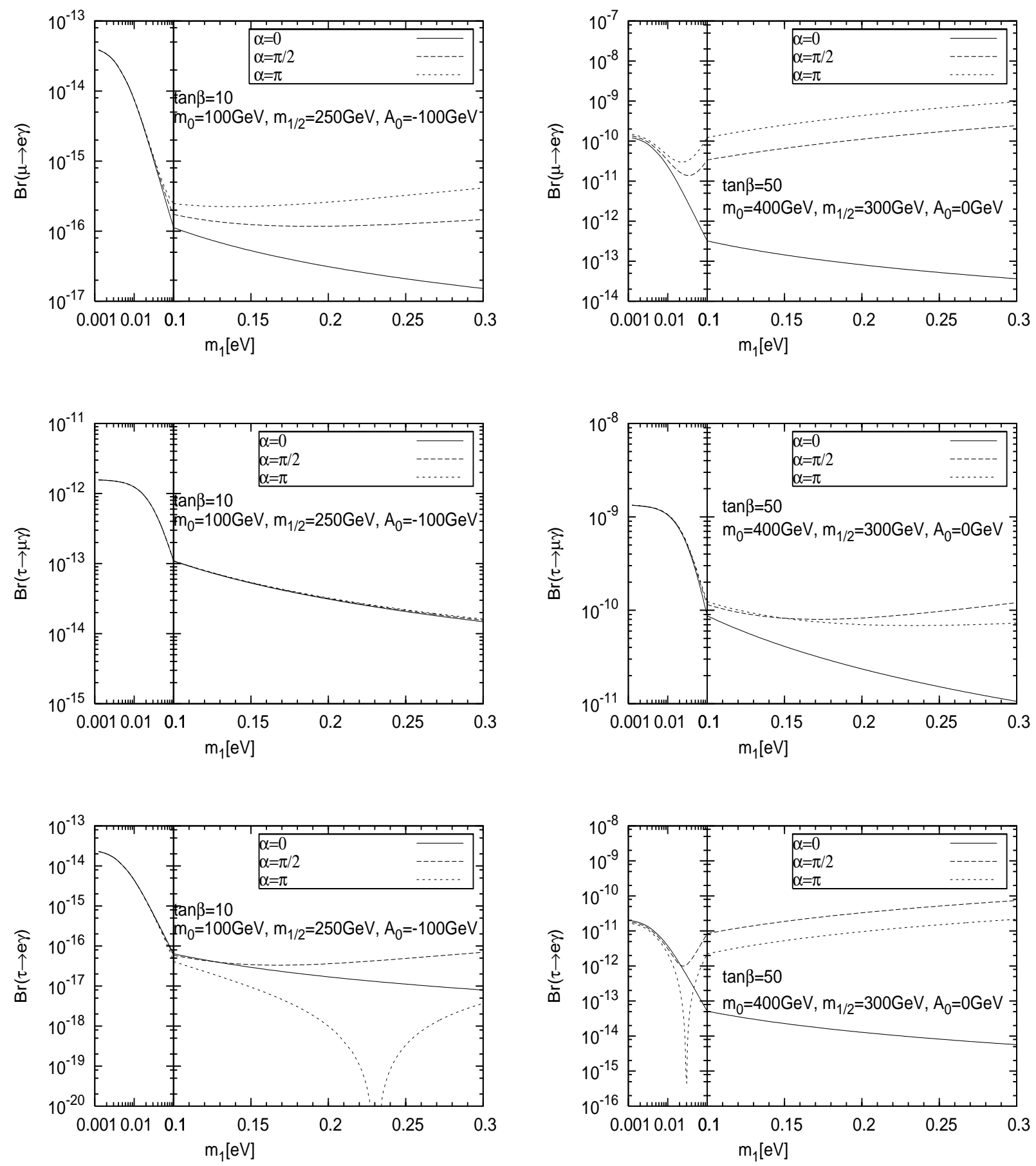

(A)

(B)

Figure 3: The branching ratios of the LFV decays $\mu \rightarrow e+\gamma, \tau \rightarrow \mu+\gamma$ and $\tau \rightarrow e+\gamma$ versus $m_{1}$ in the case of $\mathbf{R}=\mathbf{1}$ and for $\alpha=0 ; \pi / 2 ; \pi$. The results shown correspond to the following two sets of SUSY parameters: (A) $\tan \beta=10, m_{0}=100 \mathrm{GeV}, m_{1 / 2}=250$ $\mathrm{GeV}, A_{0}=-100 \mathrm{GeV}$, and (B) $\tan \beta=50, m_{0}=400 \mathrm{GeV}, m_{1 / 2}=300 \mathrm{GeV}, A_{0}=0$. The neutrino mixing parameters are the same as in Fig. [1 

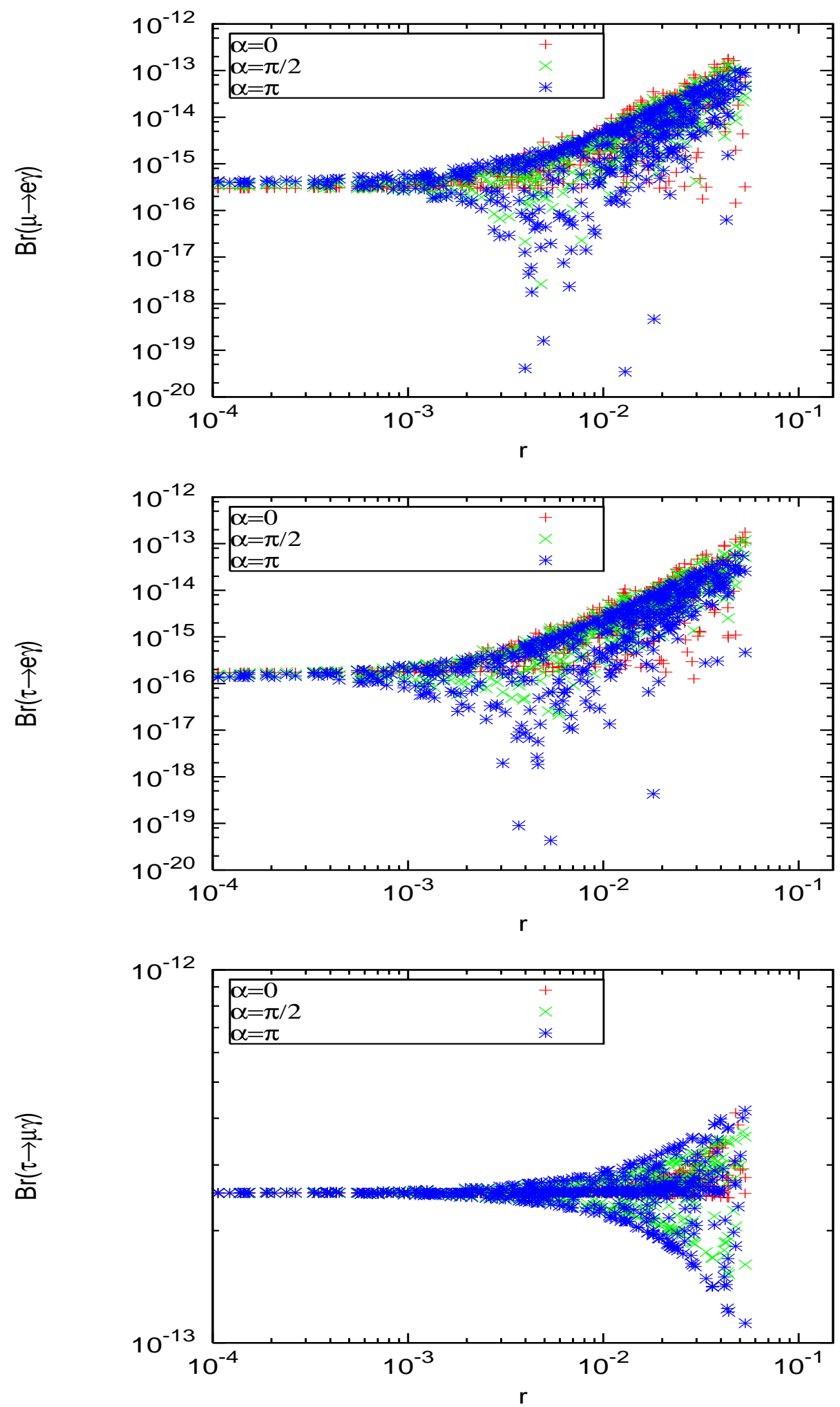

Figure 4: The branching ratios $\mathrm{BR}(\mu \rightarrow e+\gamma), \operatorname{BR}(\tau \rightarrow e+\gamma)$, and $\operatorname{BR}(\tau \rightarrow \mu+\gamma)$ as functions of $r \equiv \sqrt{a^{2}+b^{2}+c^{2}}$ for fixed $m_{1}=0.06 \mathrm{eV}, \alpha=0 ; \pi / 2 ; \pi$ and $\beta_{M}=0$. The values of the other neutrino mixing parameters at $M_{Z}$ are the same as in Fig. 1 The SUSY parameters are taken as $\tan \beta=10, m_{1 / 2}=250 \mathrm{GeV}, m_{0}=100 \mathrm{GeV}$ and $A_{0}=-100 \mathrm{GeV}$. 

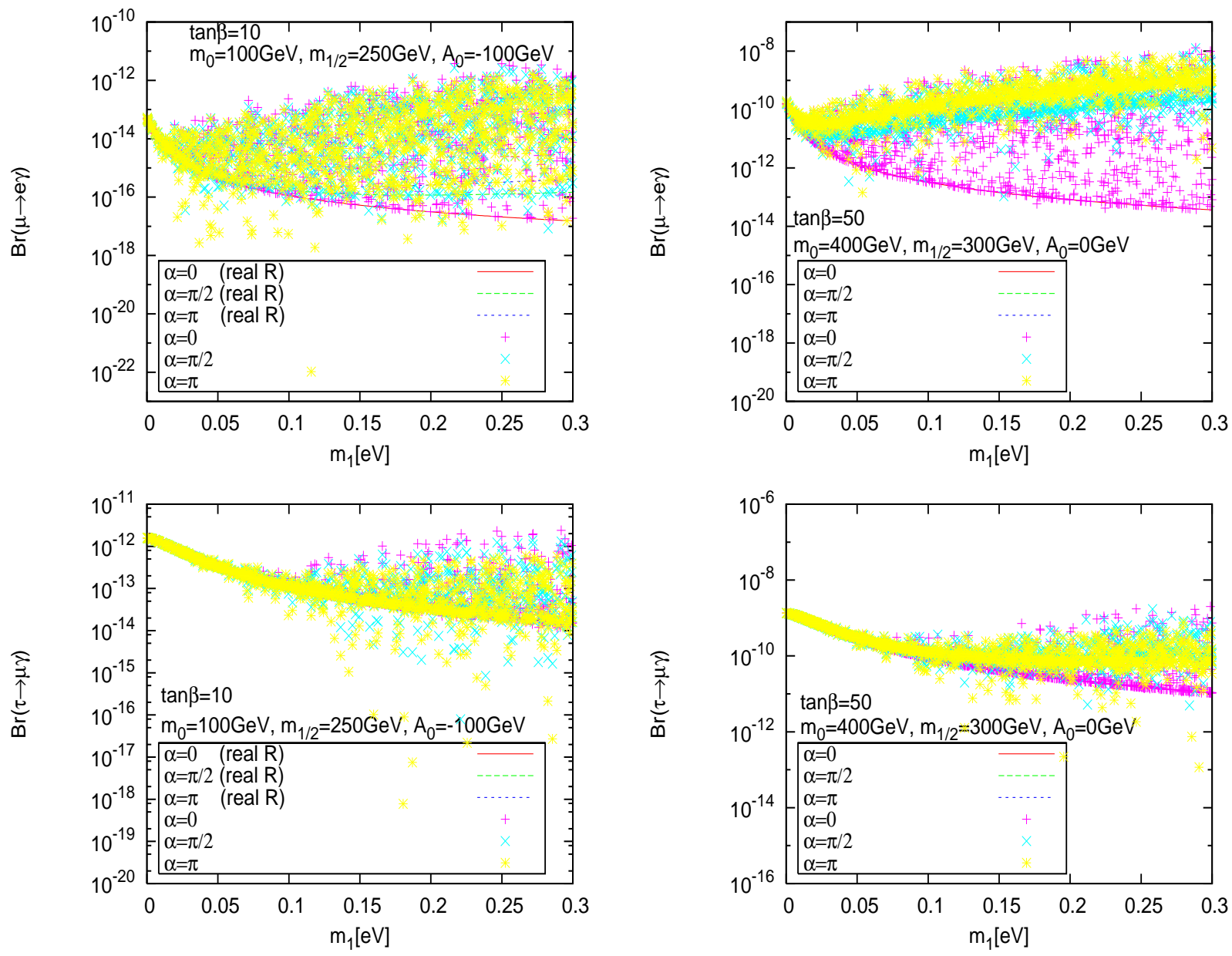

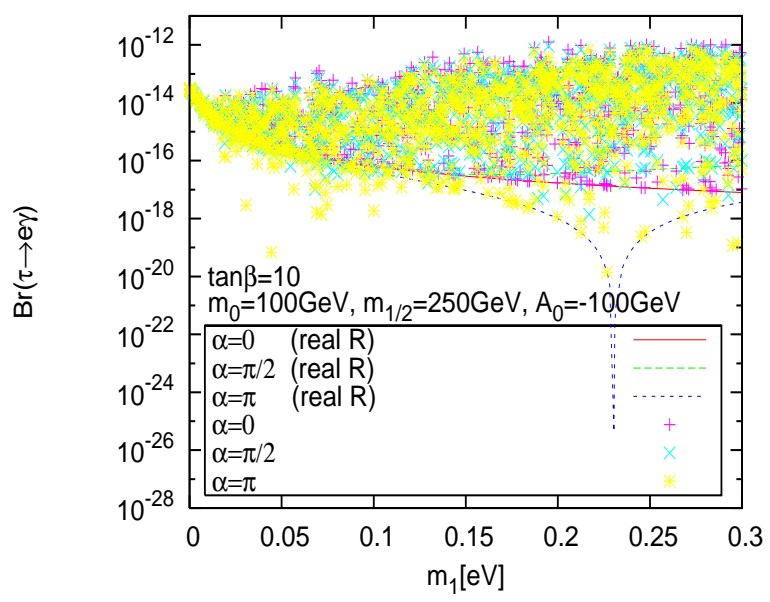

(A)

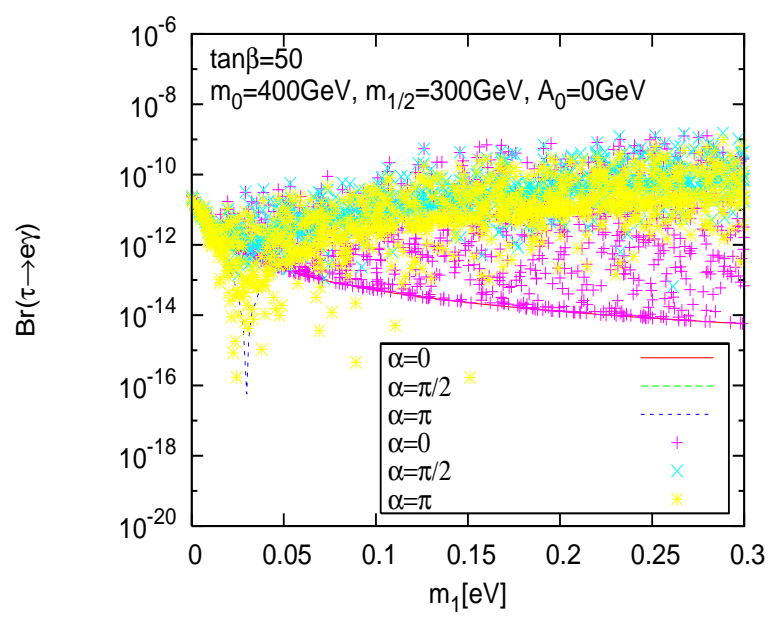

(B)

Figure 5: The same as in Fig. (3) but for complex matrix $\mathbf{R} \neq \mathbf{1}$, eq. (35.). The three constants $a, b, c$ parametrising the matrix $\mathbf{R}$ are assumed to lie in the interval $-0.1 \leq a, b, c \leq 0.1$. For comparison results for real $\mathbf{R}=\mathbf{1}$ are also shown (see text for further details). 